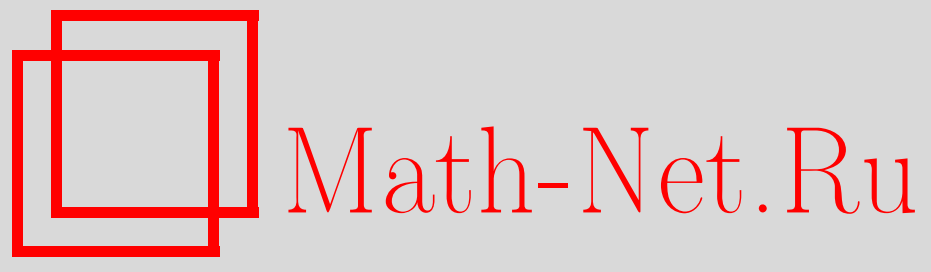

В. И. Драгович, М. Раднович, Псевдоинтегрируемые биллиарды и решетки двойных отражений, УМН, 2015, том 70, выпуск 1, 3-34

DOI: https://doi.org/10.4213/rm9648

Использование Общероссийского математического портала Math-Net.Ru подразумевает, что вы прочитали и согласны с пользовательским соглашением http://www . mathnet.ru/rus/agreement

Параметры загрузки:

IP: 54.210 .77 .194

26 апреля 2023 г., 16:29:15

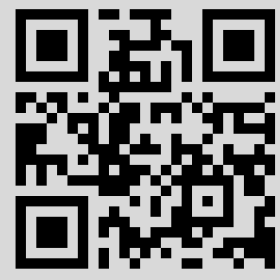




\section{Псевдоинтегрируемые биллиарды и решетки двойных отражений}

\section{В. И. Драгович, М. Раднович}

Дается обзор двух недавно возникших направлений в исследовании биллиардов в пучках квадрик: псевдоинтегрируемые биллиарды на плоскости и решетки двойных отражений.

Библиография: 48 названий.

Ключевые слова: эллиптический биллиард, многоугольный биллиард, периодические траектории, конфигурация двойного отражения, дискретные интегрируемые системы, конгруэнции прямых, псевдоинтегрируемость.

DOI: $10.4213 / \mathrm{rm} 9648$

\section{СОДЕРЖАНИЕ}

1. Введение и исторические сведения ......................... 4

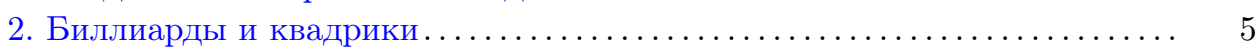

2.1. Эллиптические биллиарды и софокусные коники на плоскости . 5

2.2. Софокусные квадрики в пространстве, биллиарды и конфигу-

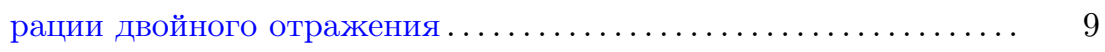

3. Псевдоинтегрируемые биллиарды . . . . . . . . . . . . . . . . . . 11

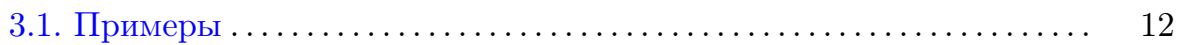

3.2. Компактные слои в фазовом пространстве ............... 15

3.3. Связь с биллиардами в прямоугольных многоугольниках ...... 19

3.4. Арифметические и эргодические свойства............... 22

4. Интегрируемые конгруэнции прямых и решетки двойных отражений . . 24

4.1. Биллиардная алгебра и квад-графы ................... 26

4.2. Решетки двойных отражений ........................ 27

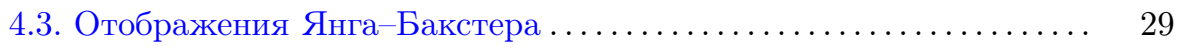

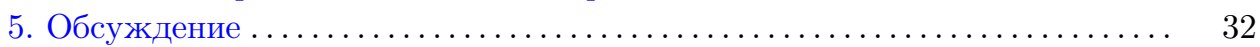

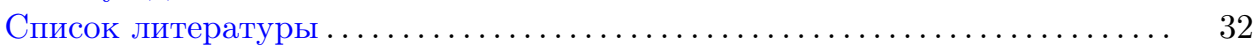

Работа выполнена при поддержке Министерства образования и науки Сербии (проект № 174020: Геометрия и топология многообразий, классическая механика и интегрируемые динамические системь) и Австралийского совета по исследованиям (грант № FL120100094).

(С) В.И. ДРАГович, М. РАДНович, 2015 


\section{1. Введение и исторические сведения}

Биллиарды в областях, ограниченных софокусными квадриками, были впервые рассмотрены Ж.-Г. Дарбу, который обобщил большую теорему Понселе на биллиарды в областях, ограниченных софокусными квадриками, в трехмерном пространстве; см. [12], [13]. Современное состояние теории таких биллиардов см. в классической книге В.В. Козлова и Д. В. Трещёва [36; гл. 4] и в книге С. Табачникова [43; гл. 4], имеющей выраженную учебную направленность. Недавно в статье [17] и в книге [18] мы представили обзор результатов, полученных в самом конце XX в. и в первой декаде XXI в. В настоящей статье нашей целью является рассказ о двух новых направлениях исследований, возникших после 2010 г.

Исследования в первом направлении представлены в разделе 3 ; они сфокусированы на плоских биллиардах, с биллиардными столами, ограниченными несколькими софокусными квадриками, причем граница содержит невыпуклые углы; см. примеры на рис. 10, 13 и 21. Начало систематическому изучению таких биллиардов было положено в [22] и [23]. Было обнаружено, что динамика соответствующих систем в чем-то напоминает интегрируемые случаи, что находит выражение в существовании каустик у траекторий. Однако оказывается, что динамика в целом все же значительно богаче и имеет новые, пока не до конца изученные свойства, отличающие ее от интегрируемых случаев. $\mathrm{K}$ примеру, хотя фазовое пространство такого биллиарда также расслаивается на инвариантные компактные поверхности, род этих поверхностей может быть больше 1. В предложении 3.4 мы вычисляем этот род через число невыпуклых углов на границе. Как ни удивительно, на фиксированной инвариантной поверхности может наблюдаться несколько режимов динамики, например, периодические режимы с разными периодами наряду с апериодическими режимами. Такую динамику мы называем псевдоинтегрируемой, вслед за заметкой [41], положившей начало этой тематике; см. также [47]. Результат типа теоремы Лиувилля-Арнольда для подобной динамики установлен в теореме 3.11. Как будет показано ниже, оказывается, что псевдоинтегрируемая динамика хорошо моделируется перекладываниями отрезков и существует естественное преобразование биллиардных столов с границами-кониками в биллиардные столы, ограниченные прямоугольными многоугольниками; см. предложение 3.13.

Вторая линия исследований, представленная в разделе 4, черпает вдохновение в теореме о двойном отражении (см. теорему 2.6, а также [10], [12], [15]). Теорема о двойном отражении является прективно-геометрическим стержнем динамики биллиардов в областях, ограниченных софокусными квадриками; она утверждает, что отражения от двух софокусных квадрик можно переставлять. Четыре прямые, получающиеся отражением друг друга в контексте теоремы о двойном отражении, образуют конфигурацию двойного отражения, играющую роль квад-уравнения для недавно открытого класса интегрируемых конгруэнций прямых, называемых решетками двойных отражений [20]. Интегрируемость возникает как следствие операционной непротиворечивости биллиардной алгебры из [16]; см. теорему 4.1 о шестиконечной звезде. Там же, в разделе 4, мы даем новое определение понятия сеток двойных отраже- 
ний с целью охватить более широкий класс систем и приводим новые примеры, связанные с обобщенной большой теоремой Понселе и псевдоинтегрируемыми биллиардами.

В следующем разделе 2 мы описываем геометрический и динамический контекст нашего изложения.

\section{2. Биллиарды и квадрики}

Мы начнем с изложения фундаментальных понятий и фактов, относящихся к семействам софокусных квадрик и соответствующим биллиардам. В п. 2.1 мы сосредоточимся на кониках и эллиптических биллиардах на плоскости, а в п. 2.2 рассмотрим случай произвольной размерности.

Более подробное изложение основных (и не только) понятий, задающих геометрический контекст этой статьи, можно найти в [6], [7], [18], [27], [42].

По поводу биллиардов в областях, ограниченных софокусными квадриками, читателю имеет смысл обратиться к [9], [10], [18], [40] и к цитируемой там литературе.

\section{1. Эллиптические биллиарды и софокусные коники на плоско-} сти. Эллиптическим биллиардом [34], [36], [43] называется динамическая система, в которой единичная точечная масса движется по инерции, т. е. с постоянной скоростью, внутри эллипса, повинуясь закону отражения на границе, так что в каждой точке отскока углы вхождения и отражения, образуемые с касательной к эллипсу, равны; см. рис. 1. Мы также предполагаем, что отскоки происходят абсолютно упруго, и пренебрегаем трением.

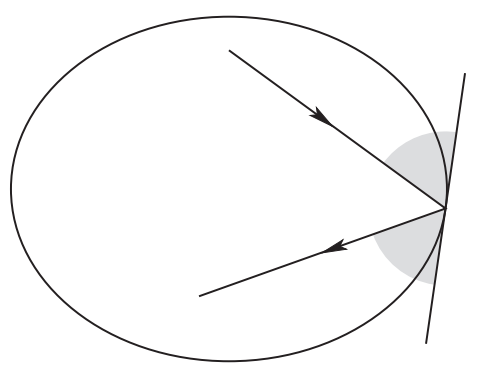

Рис. 1. Отражение от границы

Хорошо известно, что в эллиптическом биллиарде все отрезки фиксированной биллиардной траектории касаются одной и той же коники, имеющей те же фокусы, что и граница [10]; см. рис. 2.

В общем случае семейство плоских софокусных коник можно задать уравнениями

$$
\mathscr{C}_{\lambda}: \quad \frac{x^{2}}{a-\lambda}+\frac{y^{2}}{b-\lambda}=1, \quad \lambda \in \mathbb{R},
$$

где $a>b>0-$ постоянные; см. рис. 3 .

Знаменитая теорема Шаля [11] говорит, что у биллиардной траектории все отрезки касаются некоторой фиксированной коники, софокусной с границей 


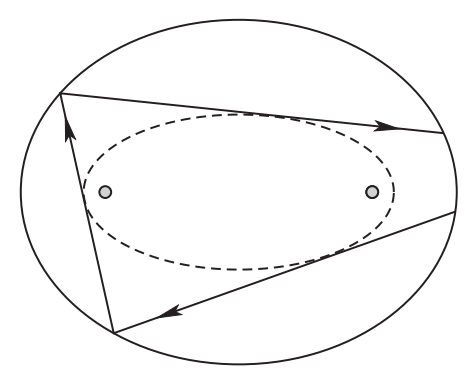

Рис. 2. Каустика биллиардной траектории

(см. также [18], [36]). Эта коника называется каустикой рассматриваемой траектории; см. рис. 2.

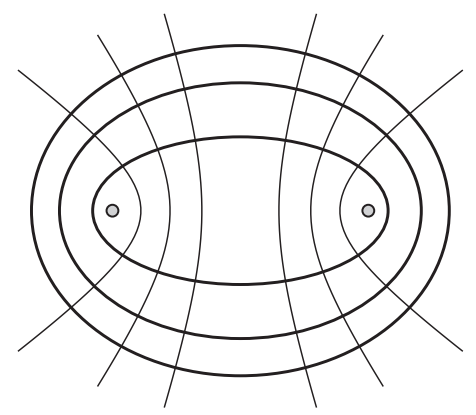

Рис. 3. Семейство софокусных коник в евклидовой плоскости

Если после $n$ отражений траектория замыкается, то по теореме Понселе любая траектория биллиардной динамической системы, имеющая ту же каустику, также периодична с периодом $n$.

\section{Мера на каустике в эллиптическом биллиарде.}

ПреДЛОЖение 2.1. Существуют мера $\mu$ на эллипсе $\mathscr{C}_{\alpha_{0}}$ и функция

$$
\rho:\left(-\infty, \alpha_{0}\right) \rightarrow \mathbb{R}
$$

со следующими свойствами:

(i) мера $\mu$ безатомарная, m.е. $\mu(\{X\})=0$ для всякой точки $X$ эллипса $\mathscr{C}_{\alpha_{0}}$;

(ii) $\mu(\ell) \neq 0$ для всякой открытой дуги $\ell$ эллипса $\mathscr{C}_{\alpha_{0}}$;

(iii) для любого $\lambda<\alpha_{0}$ и всякой тройки точек $X \in \mathscr{C}_{\lambda}, A \in \mathscr{C}_{\alpha_{0}}, B \in \mathscr{C}_{\alpha_{0}}$ такой, что $X A$ и $Х В$ касаются $\mathscr{C}_{\alpha_{0}}$, выполнено равенство

$$
\mu(A B)=\rho(\lambda)
$$

(iv) $\mu\left(\mathscr{C}_{\alpha_{0}}\right)=1$.

Отметим, что свойство (iii) означает следующее: все дуги с концами на двух касательных, выпущенных из точки на $\mathscr{C}_{\lambda}$, имеют одну и ту же длину относительно меры $\mu$; см. рис. 4 . 


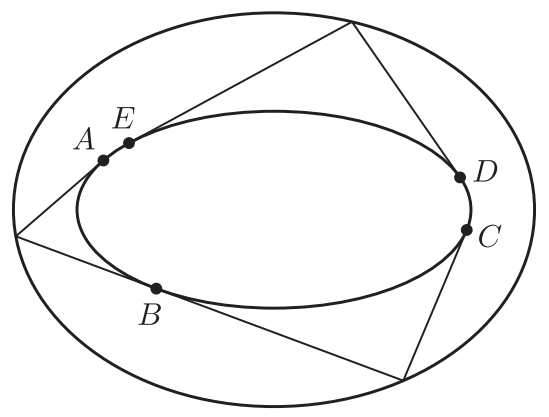

Рис. 4. $\mu(A B)=\mu(B C)=\mu(D E)$

ДокАЗАТЕЛЬСтво. По поводу конструкции такой меры см. [33], [22], а также [43], [3].

ЗАМЕЧАНИЕ 2.2. Функцию $\rho$ из предложения 2.1 называют функцией вращения, а ее значения - числами вращения. Заметим, что $\rho$ - непрерывная, строго убывающая функция с областью значений, равной интервалу $(0,1 / 2)$ :

$$
\lim _{\lambda \rightarrow-\infty} \rho(\lambda)=\frac{1}{2}, \quad \lim _{\lambda \rightarrow \alpha_{0}} \rho(\lambda)=0 .
$$

Эллиптический биллиард как гамильтонова система. Стандартная скобка Пуассона для биллиардной системы задается формулой

$$
\{f, g\}=\frac{\partial f}{\partial x} \frac{\partial g}{\partial \dot{x}}-\frac{\partial f}{\partial \dot{x}} \frac{\partial g}{\partial x}+\frac{\partial f}{\partial y} \frac{\partial g}{\partial \dot{y}}-\frac{\partial f}{\partial \dot{y}} \frac{\partial g}{\partial y} .
$$

Определим функции

$$
K_{\lambda}(x, y, \dot{x}, \dot{y})=\frac{\dot{x}^{2}}{a-\lambda}+\frac{\dot{y}^{2}}{b-\lambda}-\frac{(\dot{x} y-\dot{y} x)^{2}}{(a-\lambda)(b-\lambda)} .
$$

Они представляют хорошо известные первые интегралы биллиардной системы; см. [36].

ПреДЛОжЕНИЕ 2.3. Любъе две функиии $K_{\lambda}$ коммутируют:

$$
\left\{K_{\lambda_{1}}, K_{\lambda_{2}}\right\}=0
$$

и при $\lambda_{1} \neq \lambda_{2}$ эти функции функционально независимь.

Следующий результат доказывается непосредственно.

ПРЕДЛОЖЕНИЕ 2.4. Если в области, ограниченной произвольной коникой $\mathscr{C}_{\lambda_{0}}$, точечная масса движется со скоростью s по биллиардной траектории с каустикой $\mathscr{C}_{\alpha_{0}}$, то каждая из функций $K_{\lambda}$ сохраняет постоянное значение, равное

$$
K_{\lambda}=\frac{\alpha_{0}-\lambda}{(a-\lambda)(b-\lambda)} s^{2},
$$

вдоль этой траектории. 
СлеДСТВиЕ 2.5. В области с границей, состоящей из нескольких дуг софокусных коник, каждая функция $K_{\lambda}$ является интегралом движения биллиардной частицы.

Напомним, что гамильтонова система на $2 n$-мерном симплектическом многообразии называется вполне интегрируемой, если у нее есть $n$ функционально независимых первых интегралов, причем у любой пары этих интегралов скобка Пуассона равна нулю. Хорошо известно, что теорема Лиувилля-Арнольда (см. [3]) дает описание регулярных компактных слоев для вполне интегрируемой гамильтоновой системы, т. е. совместных множеств уровня ее первых интегралов, как торов, причем динамика на этих инвариантных торах является квазипериодической и равномерной.

Биллиарды, хотя на них и наложены односторонние связи, можно рассматривать как гамильтоновы системы. Как было показано выше, биллиард внутри эллипса можно считать вполне интегрируемой системой, поскольку у него есть $n=2$ функционально независимых коммутирующих первых интеграла. Соответствующее симплектическое многообразие является четырехмерным кокасательным расслоением плоскости. Эта система редуцируется на двумерное симплектическое многообразие прямых на плоскости, в котором роль симплектической формы играет обычная форма площади. Меру из предложения 2.1 можно связать с плоской структурой на одномерном инвариантном торе, представленном коникой-каустикой.

Инвариантные торы можно представить наглядно: область между краем биллиардного стола и каустикой, заполненная соответствующими траекториями, является проекцией таких торов. Когда каустика фиксирована, любая точка из этой области оказывается проекцией четырех точек из соответствующего множества уровня в фазовом пространстве; см. рис. 5.

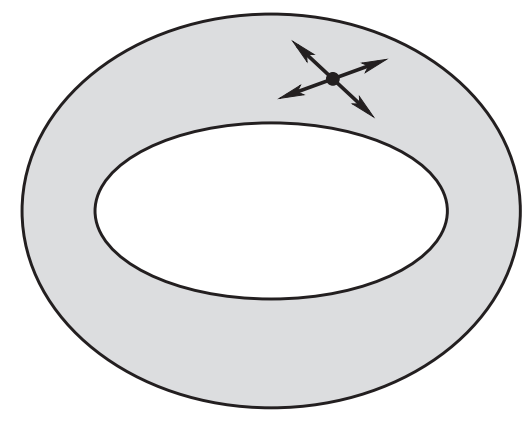

Рис. 5. Четыре возможных направления движения из данной точки при фиксированной каустике

Если каустика - эллипс, то кольцо, содержащее траектории, оказывается проекцией двух лиувиллевых торов, каждый из которых соответствует некоторому направлению обхода вокруг каустики; см. рис. 6. Если каустика - гипербола, то криволинейный четырехугольник, ограниченный ветвями гиперболы и эллипсом, является проекцией единственного тора; см. рис. 7. 


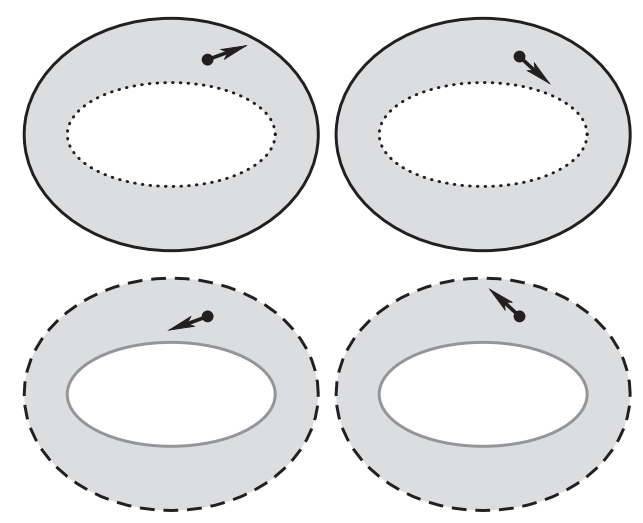

Рис. 6. Склейка колец вдоль границ, дающая два тора из фазового пространства
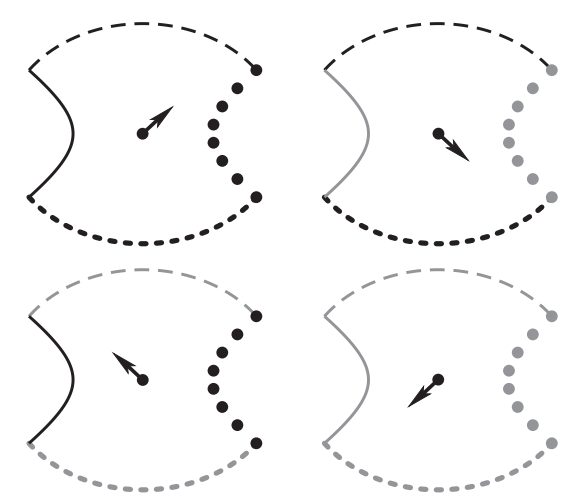

Рис. 7. Склейка криволинейных четырехугольников вдоль границ, дающая тор из фазового пространства

В разделе 3 вводится более общий класс систем - псевдоинтегрируемые системы и формулируется обобщение теоремы Лиувилля-Арнольда (см. теоремy 3.11).

2.2. Софокусные квадрики в пространстве, биллиарды и конфигурации двойного отражения. Общее семейство софокусных квадрик в $d$-мерном евклидовом пространстве задается уравнением

$$
\frac{x_{1}^{2}}{b_{1}-\lambda}+\cdots+\frac{x_{d}^{2}}{b_{d}-\lambda}=1, \quad \lambda \in \mathbb{R}
$$

где $b_{1}>b_{2}>\cdots>b_{d}>0$; см. рис. 8 .

Подобная система обладает следующими свойствами:

(а) любая точка пространства $\mathbb{E}^{d}$ является точкой пересечения ровно $d$ квадрик из (2); вдобавок все эти квадрики имеют разные геометрические типы; 


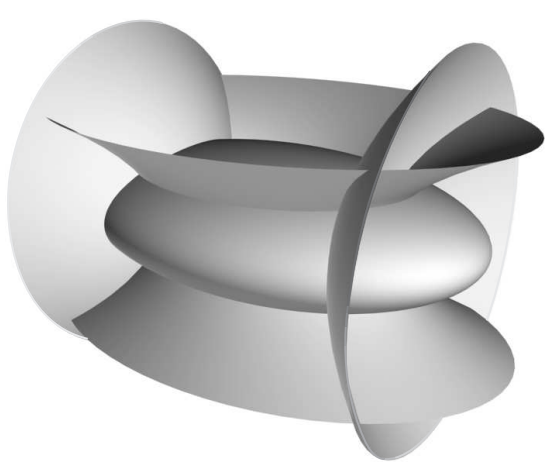

Рис. 8. Софокусные квадрики в трехмерном евклидовом пространстве

(b) семейство (2) содержит невырожденные квадрики ровно $d$ геометрических типов, каждому из которых отвечает один из непересекающихся интервалов изменения параметра $\lambda:\left(-\infty, b_{d}\right),\left(b_{d}, b_{d-1}\right), \ldots,\left(b_{2}, b_{1}\right)$;

(c) по теореме Шаля любая прямая в пространстве касается ровно $d-1$ квадрики из семейства (2);

(d) любые две прямые, связанные законом отражения от некоторой квадрики из $(2)$, касаются одних и тех же $d-1$ софокусных квадрик.

В силу последнего свойства у биллиардной траектории в области, ограниченной софокусными квадриками, есть $d-1$ каустик из софокусного семейства.

\section{Конфигурации двойного отражения.}

Теорема 2.6. Пусть $a, b, c, d$ - прямые, а $\mathscr{Q}_{\alpha}, \mathscr{Q}_{\beta}$ - собокусные квадрики в пространстве, имеющие следующие свойства (см. рис. 9):

(i) прямые а и b и прямые с и d пересекаются на $\mathscr{Q}_{\alpha}$;

(ii) прямые а и с и прямые $b$ и $d$ пересекаются на $\mathscr{Q}_{\beta}$;

(iii) касательные плоскости к квадрикам в точках пересечения принадлежат одному пучку плоскостей.

Тогда пары а, $b$ и с, d связаны законом отражения от $\mathscr{Q}_{\alpha}$, a пары $a$, с и $b, d$ удовлетворяют закону отражения от $\mathscr{Q}_{\beta}$.

ОПРЕДЕЛЕНИЕ 2.7. Мы будем говорить, что четверка прямых $a, b, c, d$, удовлетворяющих условиям теоремы 2.6, образует конфигурацию двойного отражения.

Мы представляем конфигурацию двойного отражения на рис. 9.

Теперь перечислим основные сведения, имеющиеся по конфигурациям двойного отражения.

ПреДЛОЖЕНИЕ 2.8. Пусть $a, b, c$ - прямые, $а \mathscr{Q}_{\alpha}, \mathscr{Q}_{\beta}$ - софокусные квадрики. Предположим, что а и $b$ получаются друг из друга отражением от $\mathscr{Q}_{\alpha}$, в то время как а и с получаются отражением от $\mathscr{Q}_{\beta}$. Тогда существует единственная прямая d такая, что четверка прямых $a, b, c, d$ образует конфигурацию двойного отражения. 


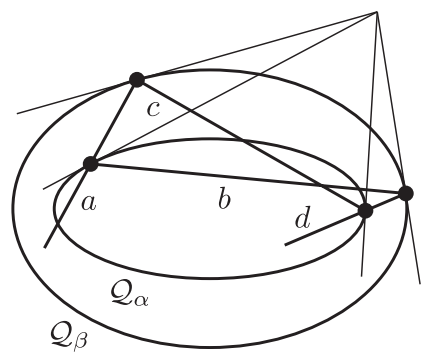

Рис. 9. Конфигурация двойного отражения

ЗАмЕчАниЕ 2.9. Как показывает предложение 2.8, конфигурация двойного отражения играет роль квад-уравнения для прямых в проективном пространстве; см. раздел 4.

\section{3. Псевдоинтегрируемые биллиарды}

В этом разделе рассматриваются биллиарды в областях, ограниченных несколькими софокусными кониками. Ряд таких примеров приведен на рис. 10.
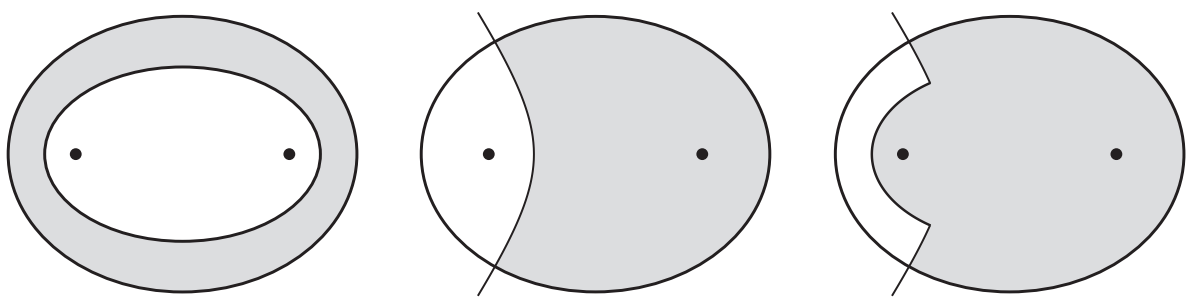

Рис. 10. Биллиардные столы, ограниченные дугами софокусных коник

Эти области ограничены кусочно гладкими кривыми, так что биллиардная траектория не определена в концах дуг, составляющих границу. Поскольку две софокусные коники (эллипс и гипербола) ортогональны в точках пересечения, концы этих дуг являются вершинами углов биллиардного стола с раствором $90^{\circ}, 270^{\circ}$ или $360^{\circ}$ : два последних угла невыпуклы, в то время как первый угол выпуклый.

Отметим, что, как показывает предельный переход от близлежащих траекторий, естественно определить отражение от вершины прямого угла так, чтобы входящий и отраженный отрезки совпали; см. рис. 11.

С другой стороны, когда касательные образуют невыпуклый угол, как на рис. 12, такого предела не существует и отражение определить нельзя. Когда биллиардный шар попадает в вершину такого угла, мы считаем, что его движение на этом заканчивается.

При исследовании биллиардов в областях с невыпуклыми углами на границе (см. [38], [48]) особый интерес представляют траектории, начинающиеся или 


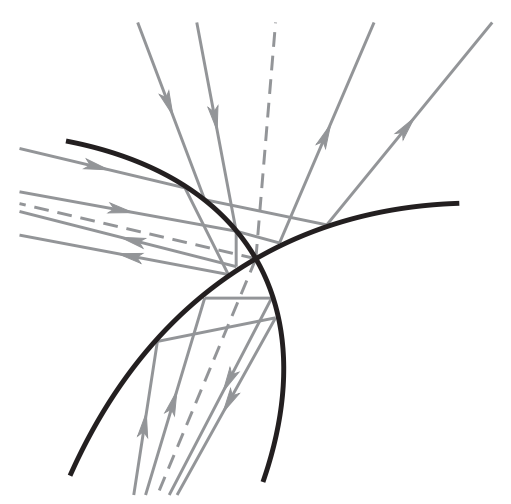

Рис. 11. Отражение от прямого угла

заканчивающиеся в вершине невыпуклого угла. Они называются сепаратрисами. Сепаратриса, оба конца которой лежат в вершинах невыпуклых углов, называется седловой связкой. Седловая связка, концы которой совпадают, называется гомоклинической петлей. Прочие биллиардные траектории, никогда не попадающие в вершину невыпуклого угла, называются регулярными.
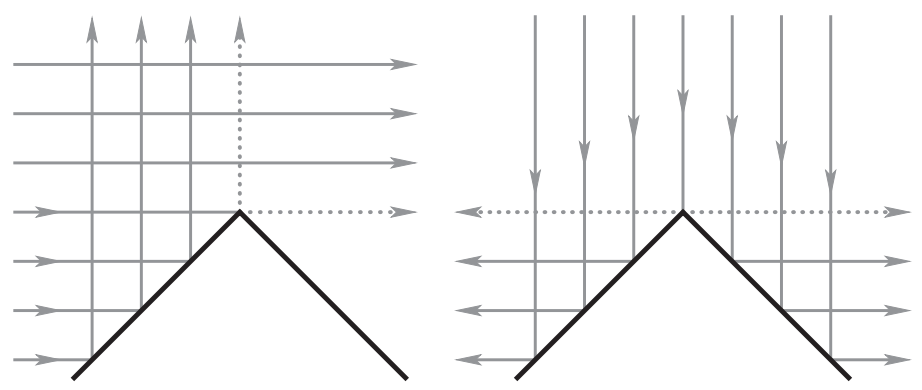

Рис. 12. Отражение от невыпуклого угла

Знакомство со свойствами динамики биллиардов в областях, граница которых состоит из нескольких софокусных коник и содержит невыпуклые углы, мы начнем в п. 3.1 с нескольких примеров. В п. 3.2 мы вернемся к общему случаю. Там будет установлено соотношение между числом невыпуклых углов на границе, родом слоев в фазовом пространстве и числом седловых связок (см. предложение 3.4 и следствие 3.7). Затем будет сформулирован аналог теоремы Лиувилля-Арнольда (см. теорему 3.11). В п. 3.3 строится отображение, трансформирующее семейство софокусных коник в систему прямых и сохраняющее биллиардные траектории с фиксированной каустикой. В п. 3.4 будут установлены связи с перекладыванием отрезков.

3.1. Примеры. Начнем с примеров, в которых семейство софокусных коник предполагается вырожденным, т. е. состоящим из концентрических окружностей и прямых, проходящих через их общий центр. Эти примеры достаточно 

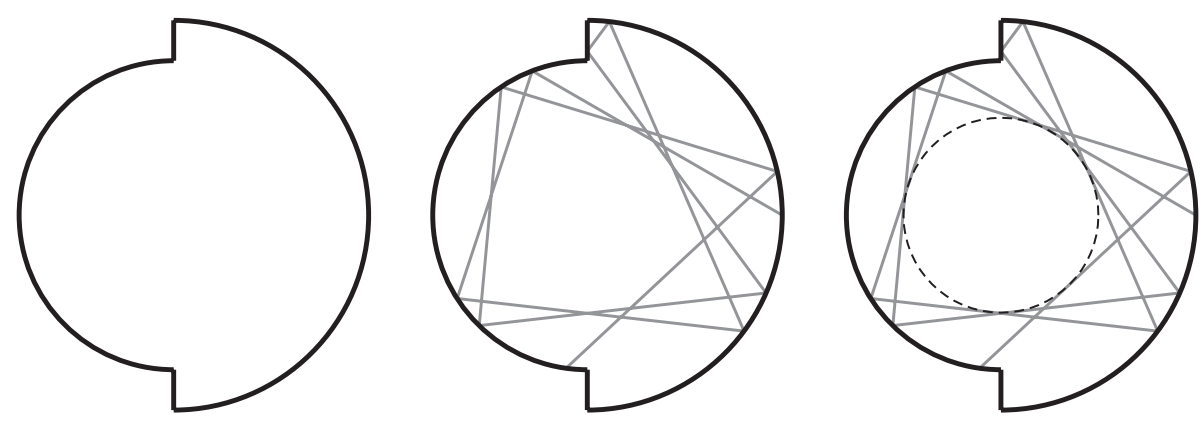

Рис. 13. Биллиардный стол, траектория и ее каустика

просты для анализа элементарными средствами; тем не менее в них реализуется все многообразие явлений, возникающих в общем, невырожденном случае.

Рассмотрим биллиардную систему в области, ограниченной двумя концентрическими полуокружностями и отрезками одного и того же диаметра, как на рис. 13. У любой траектории на таком столе имеется каустика с тем же центром, что и у полуокружностей, входящих в границу. Зафиксировав каустику, через $\rho_{1}$ и $\rho_{2}$ обозначим числа вращения биллиардов внутри граничных окружностей.

ПримеР 3.1. При $\rho_{1}=1 / 3$ и $\rho_{2}=1 / 4$ имеется 6 седловых связок, показанных на рис. 14. Заметим, что каждая из трех ломаных на рис. 14 соответствует двум траекториям, отвечающим разным направлениям движения.
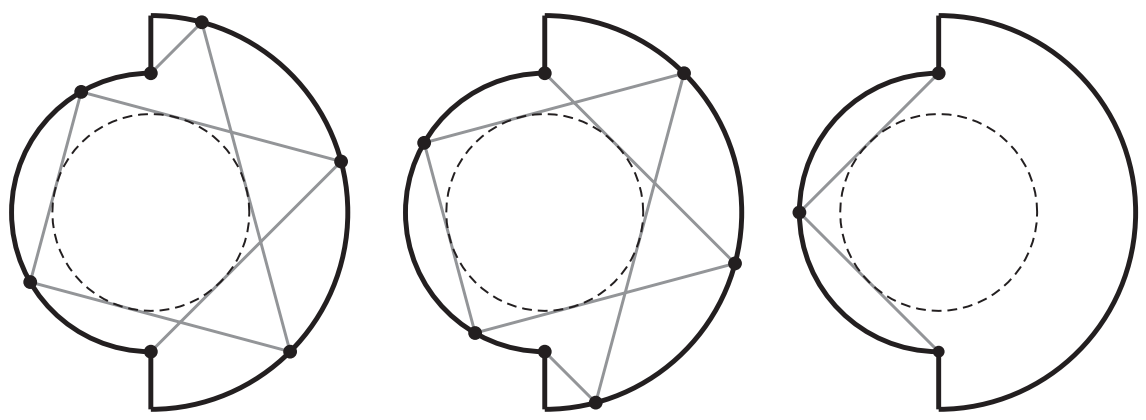

Рис. 14. Седловые связки, соответствующие числам вращения $1 / 3$ и $1 / 4$

Вершины седловых связок разбивают край биллиардного стола на 13 частей, группирующихся в два подмножества; см. рис. 15. Для траектории с фиксированной каустикой имеет место следующая альтернатива:

- либо все точки отскока попадают в серые части края, и тогда биллиардная частица по два раза отскакивает от каждой из серых частей, пока траектория не замкнется, так что траектория имеет период 12 ;

- либо все точки отскока попадают в черные части края, и тогда биллиардная частица делает один отскок от каждой из частей, пока траектория не замкнется, так что траектория имеет период 7 . 

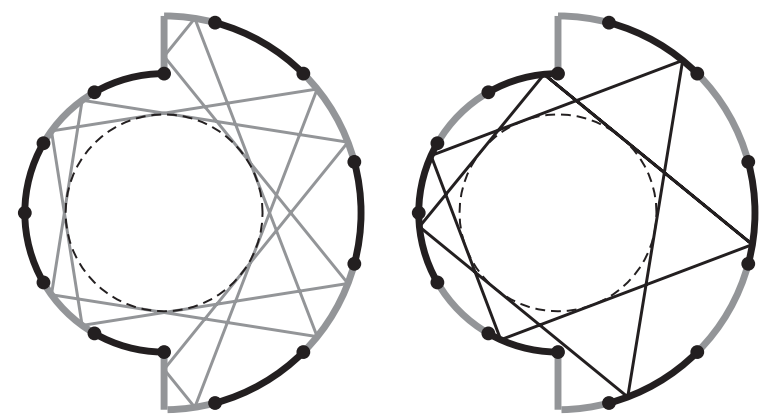

Рис. 15. Траектории с периодом 12 и 7; разбиение границы на два подмножества

ПримеР 3.2. При $\rho_{1}=(5-\sqrt{5}) / 10$ и $\rho_{2}=\sqrt{5} / 10$ имеется шесть седловых связок, четыре из которых - гомоклинические петли; см. рис. 16. Вершины
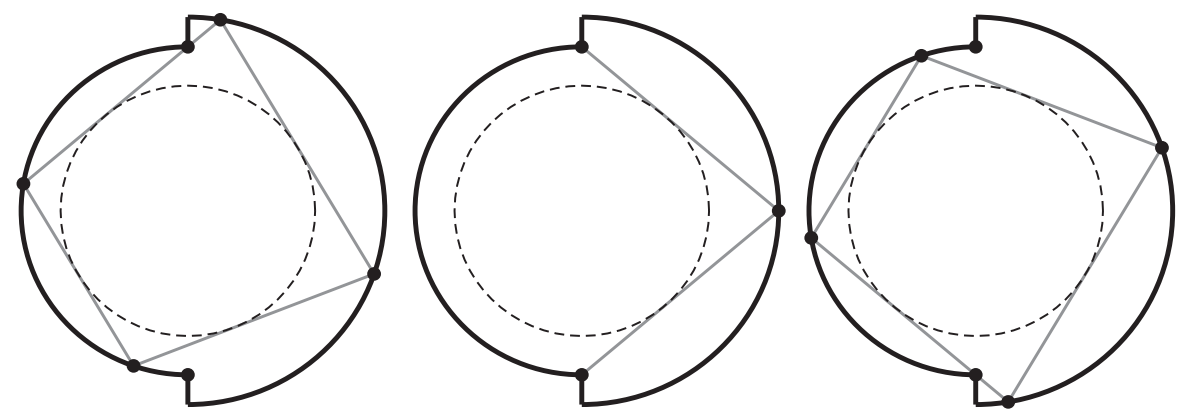

Рис. 16. Седловые связки, соответствующие числам вращения (5 $\sqrt{5}) / 10$ и $\sqrt{5} / 10$

седловых связок разбивают край стола на 11 частей, группирующихся в два подмножества; см. рис. 17.
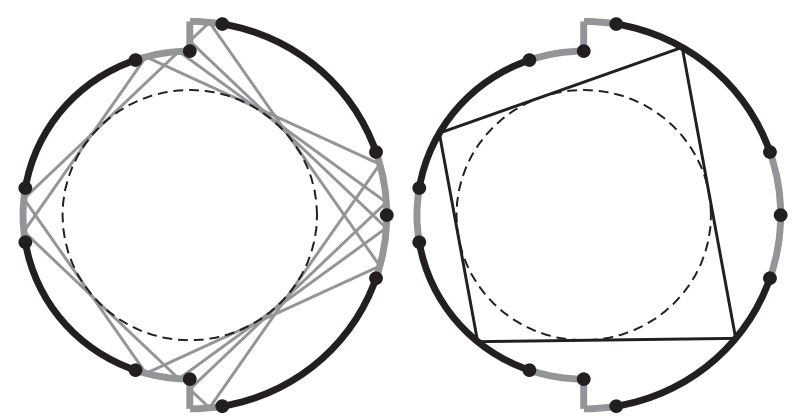

Рис. 17. Периодические траектории, отвечающие окружностям с числами вращения $(5-\sqrt{5}) / 10$ и $\sqrt{5} / 10$ 

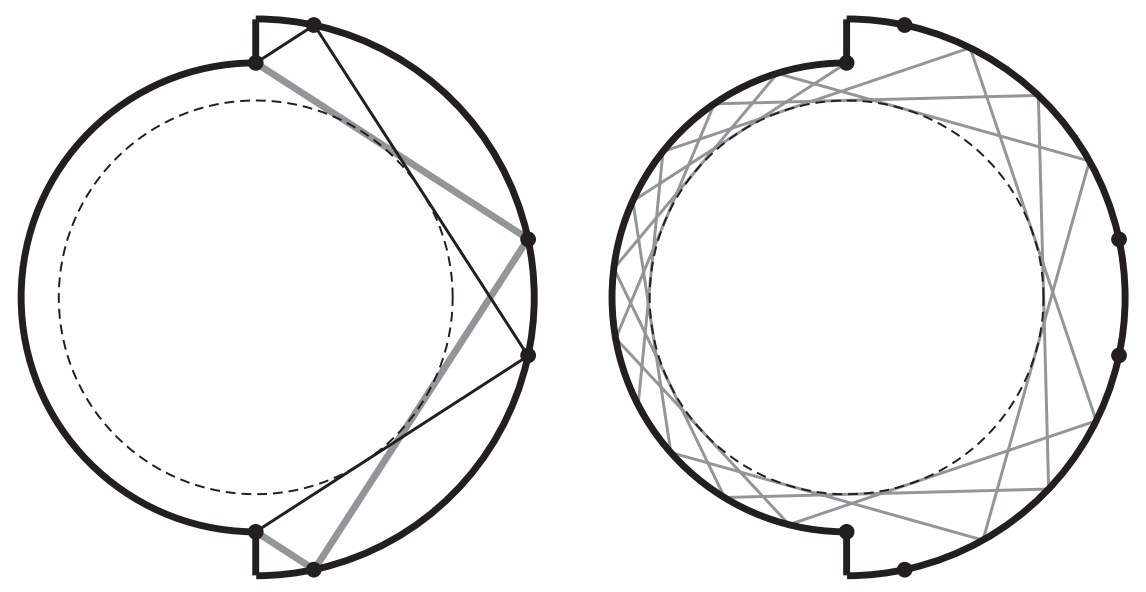

Рис. 18. Седловые связки и несколько начальных отрезков бесконечной сепаратрисы, соответствующие числам вращения $1 / 4$ и $1 / \sqrt{30}$

Для любой траектории с данной каустикой имеет место следующая альтернатива:

- либо все точки отскока попадают в серые части края, и тогда биллиардная частица два раза отскакивает от каждой из серых частей, пока траектория не замкнется; траектория имеет период 14;

- либо все точки отскока попадают в черные части края, и тогда биллиардная частица один раз отскакивает от каждой части, пока траектория не замкнется, так что траектория имеет период 4.

ПримеР 3.3. При $\rho_{1}=1 / 4$ и $\rho_{2}=1 / \sqrt{30}$ имеется 8 сепаратрис, 4 из которых являются седловыми связками. Седловые связки и одна бесконечная сепаратриса изображены на рис. 18. Прочие сепаратрисы получаются изменением направления движения или симметрией относительно горизонтальной оси. Вершины седловых связок разбивают край биллиардного стола на шесть частей, группирующихся в два подмножества (см. рис. 19). Для всякой траектории с данной каустикой имеет место следующая альтернатива:

- либо все точки отскока попадают в серые части края, и тогда траектория не является периодической;

- либо все точки отскока попадают в черные части края, и тогда частица дважды отскакивает от каждой из частей, пока не замкнется, так что траектория имеет период 6 .

Интересно было бы найти общее соответствие между числами вращения $\rho_{1}, \rho_{2}$ и поведением биллиардных траекторий [24].

3.2. Компактные слои в фазовом пространстве. Пусть $\mathscr{D}$ - ограниченная область на плоскости с границей $\Gamma=\partial \mathscr{D}$, являющейся объединением конечного числа дуг софокусных коник из семейства (1). 

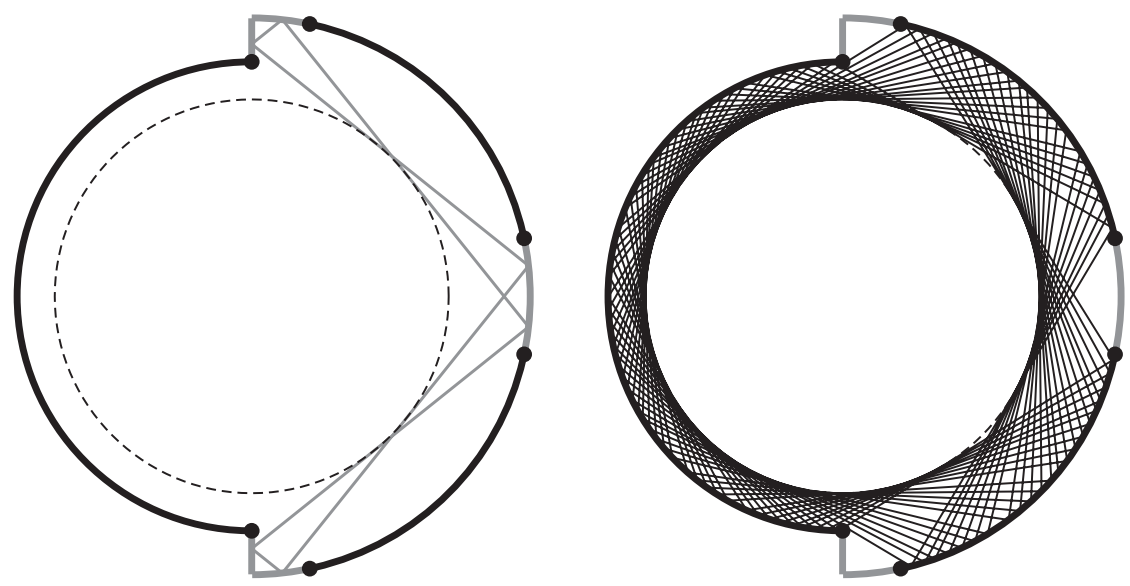

Рис. 19. Для чисел вращения $1 / 4$ и $1 / \sqrt{30}$ имеются как периодические, так и апериодические траектории

Рассмотрим биллиардную систему в $\mathscr{D}$. У всякой биллиардной траектории есть каустика - коника из семейства (1), касающаяся всех прямых, содержащих отрезки траектории; см. рис. 20. Зафиксируем $\mathscr{C}_{\alpha_{0}}$ в качестве каустики.
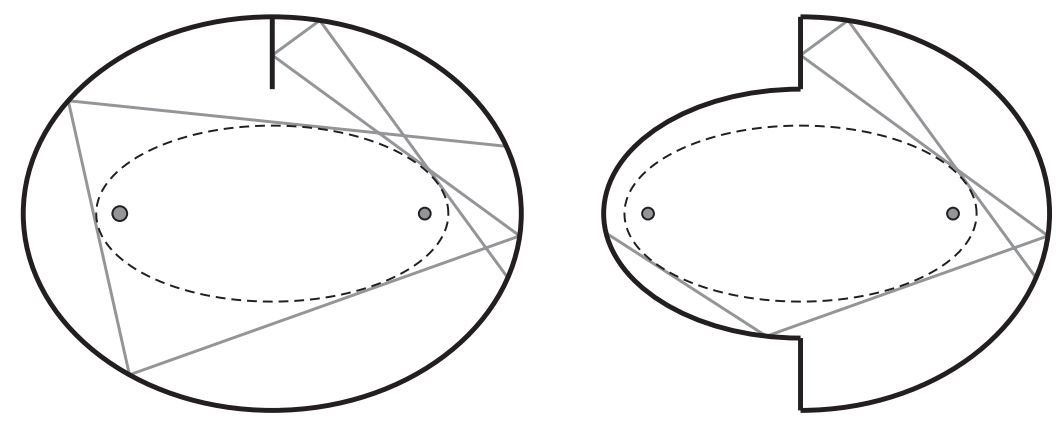

Рис. 20. Каустики биллиардных траекторий

Заметим, что касательные к конике заполняют бесконечную область на плоскости: если коника - эллипс, то эта область является его внешностью, а для гиперболы это - часть плоскости между ветвями гиперболы.

Обозначим через $\mathscr{D}_{\alpha_{0}}$ пересечение $\mathscr{D}$ с областью, заполненной касательными к $\mathscr{C}_{\alpha_{0}}$. Все биллиардные траектории с каустикой $\mathscr{C}_{\alpha_{0}}$ лежат в $\mathscr{D}_{\alpha_{0}}$, которое представляет собой ограниченное множество с границей $\Gamma_{\alpha_{0}}=\partial \mathscr{D}_{\alpha_{0}}$, являющейся объединением конечного числа дуг коник из семейства (1).

Будем предполагать, что $\mathscr{D}_{\alpha_{0}}$ также связно. В противном случае будем рассматривать одну из компонент связности этого множества.

Биллиардные траектории в $\mathscr{D}$, имеющие каустику $\mathscr{C}_{\alpha_{0}}$, отвечают некоторому компактному слою $\mathscr{M}_{\alpha_{0}}$, который можно получить склейкой 4 экземпляров $\mathscr{D}_{\alpha_{0}}$ вдоль соответствующих дуг границы $\Gamma_{\alpha_{0}}=\partial \mathscr{D}_{\alpha_{0}}$, как объяснялось в п. 2.1. 
ПреДЛОЖенИЕ 3.4. Рассмотрим биллиард в $\mathscr{D}$ с каустикой $\mathscr{C}_{\alpha_{0}}$. Если у соответствующей области $\mathscr{D}_{\alpha_{0}}$ на границе $\Gamma_{\alpha_{0}}$ имеется $r$ невыпукльх углов, то род $\mathscr{M}_{\alpha_{0}}$ равен $r+1$.

ДокАзАтЕльство. По формуле Эйлера-Пуанкаре сумма кратностей всех седел равна $2 g-2$. Седла в $\mathscr{M}_{0}$ соответствуют полным углам и углам раствора $270^{\circ}$ - это вытекает из того факта, что в вершину полного угла проецируются две седловые тоски кратности 1 , а в вершину угла раствора $270^{\circ}$ проецируется одна седловая точка кратности 2. Предложение доказано.

ПримеР 3.5. (i) Если на границе нет невыпуклых углов, так что $k=0$, то $\mathscr{M}_{\alpha_{0}}-$ тор.

(ii) Если на границе ровно один невыпуклый угол, неважно, имеет ли он раствор $270^{\circ}$ или является полным (см. рис. 21), то $g=2$.

(iii) В примерах из п. 3.1 род соответствующего слоя слоения в фазовом пространстве равен 3 .
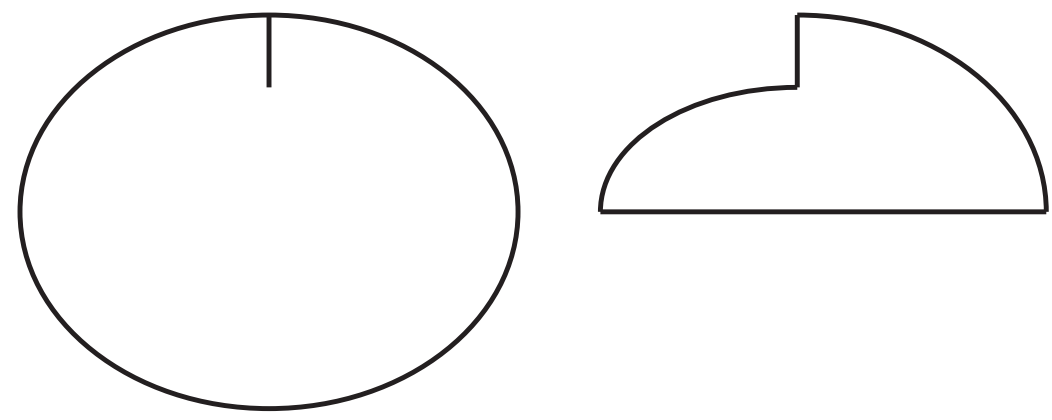

Рис. 21. Биллиардные столы с одним невыпуклым углом на краю

Аналогично [46] получаем следующую оценку общего числа $N=N\left(\mathscr{M}_{\alpha_{0}}\right)$ седловых связок.

ПРЕДЛОЖЕНИЕ 3.6. Общее число $N=N\left(\mathscr{M}_{\alpha_{0}}\right)$ одновременно существующих седловых связок ограничено сверху:

$$
N\left(\mathscr{M}_{\alpha_{0}}\right) \leqslant \frac{1}{2} \sum_{i=1}^{k} s_{i}=k-\chi\left(\mathscr{M}_{\alpha_{0}}\right),
$$

где $k$ - число особъх точек потока на $\mathscr{M}_{\alpha_{0}}, s_{1}, \ldots, s_{k}$ - число сепаратрис в каждой из особых точек, а ұ - эйлерова характеристика.

В качестве следствия получаем следующий результат.

СледСтвие 3.7. Рассмотрим биллиард в $\mathscr{D}$ с каустикой $\mathscr{C}_{\alpha_{0}}$. Если на границе $\Gamma_{\alpha_{0}}$ соответствующей области $\mathscr{D}_{\alpha_{0}}$ имеется $r$ невыпукльх углов, то $N \leqslant 3 r$.

ПримеР 3.8. Во всех примерах из п. 3.1 имеем $r=2$, так что число седловых связок в них имеет верхнюю оценку $6=3 r$. В примерах 3.1 и 3.2 имеется шесть седловых связок, а в примере 3.3 - четыре связки. 
Как хорошо известно, теорема Лиувилля-Арнольда (см. [3]) описывает регулярные компактные слои вполне интегрируемой гамильтоновой системы как торы с квазипериодической динамикой на этих инвариантных торах. На некоторых из них динамика чисто периодическая, с некоторым фиксированным периодом.

В отличие от вполне интегрируемых гамильтоновых систем, в предложении 3.4 мы показали, что компактные слои для наших биллиардов могут иметь род, больший 1. Вдобавок, как видно на примерах из п. 3.1, на одном и том же листе могут быть траектории с разными периодами и даже периодические и апериодические траектории одновременно.

Мы используем теорему Майера из теории измеримых слоений (см. [37], [46]), чтобы дать аналог теоремы Лиувилля-Арнольда для псевдоинтегрируемых биллиардных систем - теорему 3.11. Сначала нам нужно ввести измеримое слоение для рассматриваемых здесь биллиардов.

Теорема 3.9. Измеримое слоение задается ядром замкнутой 1-формы

$$
w=\frac{d \lambda_{1}}{\sqrt{\left(a-\lambda_{1}\right)\left(b-\lambda_{1}\right)\left(\alpha_{0}-\lambda_{1}\right)}}+\frac{d \lambda_{2}}{\sqrt{\left(a-\lambda_{2}\right)\left(b-\lambda_{2}\right)\left(\alpha_{0}-\lambda_{2}\right)}},
$$

где $\lambda_{1}, \lambda_{2}$ - координаты Якоби, соответствующие софокусному семейству (1).

ДокАзАтельство. В эллиптических координатах касательные прямые к $\mathscr{C}_{\alpha_{0}}$ описываются дифференциальным уравнением [29], [30]

$$
\frac{d \lambda_{1}}{\sqrt{\left(a-\lambda_{1}\right)\left(b-\lambda_{1}\right)\left(\alpha_{0}-\lambda_{1}\right)}}+\frac{d \lambda_{2}}{\sqrt{\left(a-\lambda_{2}\right)\left(b-\lambda_{2}\right)\left(\alpha_{0}-\lambda_{2}\right)}}=0 .
$$

1-форма $w$ замкнута, как и любая форма вида $f\left(\lambda_{1}\right) d \lambda_{1}+g\left(\lambda_{2}\right) d \lambda_{2}$. Теорема доказана.

ЗАмЕчАниЕ 3.10. Знаки квадратных корней в (3) зависят от листа накрытия области $\mathscr{D}_{\alpha_{0}}$ многообразием $\mathscr{M}_{\alpha_{0}}$ и от квадранта координатной плоскости. Первое слагаемое меняет знак, когда при пересечении эллипса мы переходим на другой лист накрытия или когда мы пересекаем вырожденный эллипс из (1), являющийся отрезком с концами в фокусах. Второе слагаемое меняет знак, когда при пересечении гиперболы мы переходим на другой лист накрытия, либо при пересечении вырожденной гиперболы из семейства (1).

ТЕОРема 3.11. Существуют попарно непересекающиеся открытые подобласти $D_{1}, \ldots, D_{n}$ слоя $\mathscr{M}_{\alpha_{0}}$, каждая из которых инвариантна относительно биллиардного потока и замыкания которых покрывают $\mathscr{M}_{\alpha_{0}}$, причем для $j \in\{1, \ldots, n\}$ имеет место следующая альтернатива:

- либо $D_{j}$ coстоит из периодических биллиардных траекторий и гомеоморфна иилиндру;

- либо $D_{j}$ состоит из апериодических биллиардных траекторий, каждая из которых плотна в $D_{j}$.

Граница каждой области $D_{j}$ образована седловыми связками. 
Во всех примерах из п. 3.1 седловые связки разбивают лист слоения на три несвязные подобласти.

Пример 3.1. Одна из областей содержит все траектории с периодом 12 , а ее граница состоит из всех четырех седловых связок с семью вершинами. Две области содержат траектории с периодом 7: каждая из них соответствует одному из направлений обхода вокруг центра. Граница подобной области образована тремя седловыми связками, закручивающимися в одном и том же направлении.

Пример 3.2. Одна из областей содержит все траектории с периодом 14 , а ее граница состоит из всех шести седловых связок. Две области содержат траектории с периодом 4: каждая из них соответствует одному из направлений обхода вокруг центра. Граница подобной области состоит из двух седловых связок с пятью вершинами каждая, которые закручиваются в одном и том же направлении.

Пример 3.3. Одна из областей содержит все периодические траектории, а ее граница состоит из всех четырех седловых связок. Две области содержат апериодические траектории: каждая из них соответствует одному из направлений обхода вокруг центра. Граница подобной области состоит из двух седловых связок, которые закручиваются в одном и том же направлении.

Динамические системы с многозначными интегралами изучались несколькими авторами; упомянем здесь [4], [35] [39]. Термин "псевдоинтегрируемые системы" восходит к началу 1980-х годов; см. [41].

3.3. Связь с биллиардами в прямоугольных многоугольниках. Зафиксируем эллипс $\mathscr{C}_{\alpha_{0}}$ из семейства $(1)$. Через $\mathscr{C}_{\alpha_{0}}^{\text {ext }}$ обозначим внешность эллипса в $\mathbb{R}^{2}$ :

$$
\mathscr{C}_{\alpha_{0}}^{\text {ext }}=\left\{(x, y) \in \mathbb{R}^{2} \mid \frac{x^{2}}{a-\alpha_{0}}+\frac{y^{2}}{b-\alpha_{0}} \geqslant 1\right\} .
$$

В терминах эллиптических координат $\mathscr{C}_{\alpha_{0}}^{\text {ext }}$ - множество точек плоскости, первая координата Якоби которых удовлетворяет условию $\lambda_{1} \leqslant \alpha_{0}$.

Введем отображения $\sigma_{1}$ и $\sigma_{2}$ :

$$
\sigma_{i}(A)=\int_{M_{0}}^{A} \frac{d \lambda_{i}}{\sqrt{\left(a-\lambda_{i}\right)\left(b-\lambda_{i}\right)\left(\alpha_{0}-\lambda_{i}\right)}}, \quad i \in\{1,2\} .
$$

Здесь $M_{0}$ - фиксированная точка в $\mathscr{C}_{\alpha_{0}}^{\mathrm{ext}}$. Мы возьмем $M_{0} \in \mathscr{C}_{\alpha_{0}}$, чтобы в дальнейшем получить несколько более простое выражение. Далее, квадратный корень меняет знак, как только путь интегрирования пересекает соответствующую вырожденную квадрику из софокусного семейства. Но здесь мы никогда не пересекаем вырожденный эллипс, т. е. отрезок, соединяющий фокусы, так что квадратный корень

$$
\sqrt{\left(a-\lambda_{1}\right)\left(b-\lambda_{1}\right)\left(\alpha_{0}-\lambda_{1}\right)}
$$

всюду положителен. Вырожденные гиперболы лежат на осях координат, так что для корня

$$
\sqrt{\left(a-\lambda_{2}\right)\left(b-\lambda_{2}\right)\left(\alpha_{0}-\lambda_{2}\right)}
$$

мы выбираем положительные значения в первом и третьем квадрантах и отрицательные во втором и четвертом. 
Лемма 3.12. Отображение $\sigma_{1}$ определено корректно, т.е. его значение не зависит от пути интегрирования. Значение $\sigma_{2}$ зависит от числа оборотов вокруг $\mathscr{C}_{\alpha_{0}}$, совершенных путем, так что оно определено с точностъю до слагаемого вида

$$
4 \int_{b}^{a} \frac{d \lambda}{\sqrt{(a-\lambda)(b-\lambda)\left(\alpha_{0}-\lambda\right)}}
$$

Эта лемма вытекает из замкнутости 1-формы (3).

Определим теперь следующее отображение из $\mathscr{C}_{\alpha_{0}}^{\text {ext }}$ в цилиндр $\mathbb{R} / p \mathbb{Z} \times \mathbb{R}$ :

$$
\sigma(A)=\left(\int_{M_{0}}^{A} \frac{d \lambda_{2}}{\sqrt{\left(a-\lambda_{2}\right)\left(b-\lambda_{2}\right)\left(\alpha_{0}-\lambda_{2}\right)}}, \int_{M_{0}}^{A} \frac{d \lambda_{1}}{\sqrt{\left(a-\lambda_{1}\right)\left(b-\lambda_{1}\right)\left(\alpha_{0}-\lambda_{1}\right)}}\right)
$$

где

$$
p=4 \int_{b}^{a} \frac{d \lambda}{\sqrt{(a-\lambda)(b-\lambda)\left(\alpha_{0}-\lambda\right)}}
$$

ПРЕДЛОЖЕНИЕ 3.13. Отображение б обладает следующими свойствами (см. puc. 22):

(i) б отображает эллипсы из семейства (1) в отрезки прямых, параллельных оси $x$;

(ii) $\sigma$ отображает каждую из четырех дуг всякой гиперболь из (1), лежащих в $\mathscr{C}_{\alpha_{0}}^{\mathrm{ext}}$, в отрезок, параллельный оси $y$;

(iii) $\sigma$ - непрерьвное биективное отображение из $\mathscr{C}_{\alpha_{0}}^{\operatorname{ext}}$ в $\mathbb{R} / p \mathbb{Z} \times(-p / 4,0]$;
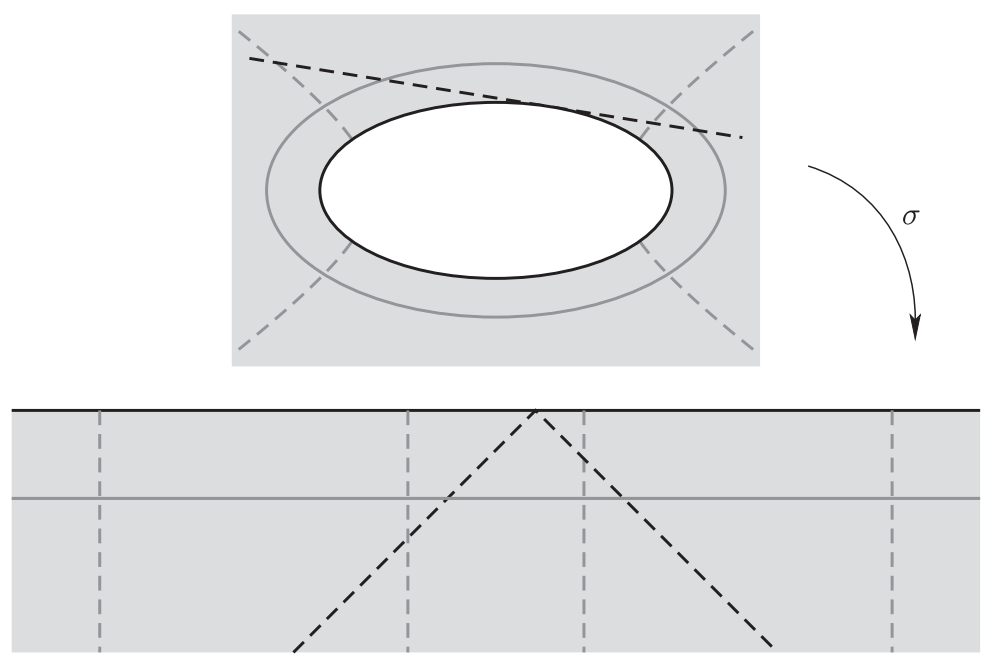

Рис. 22. Отображение $\sigma$ 
(iv) любая касательная $\kappa \mathscr{C}_{\alpha_{0}}$ отображается в объединение двух отрезков прямых с общим концом на оси $x$, каждый из которых образует углы в $45^{\circ}$ с осями координат.

ДокАЗАтельство. Поскольку $M_{0} \in \mathscr{C}_{\alpha_{0}}$, эллипс $\mathscr{C}_{\alpha_{0}}$ отображается в ось $x$. Для всякой точки $A \in \mathscr{C}_{\alpha_{0}}^{\text {ext }}$ имеем

$$
0 \geqslant \sigma_{1}(A)>\int_{\alpha_{0}}^{-\infty} \frac{d \lambda}{\sqrt{(a-\lambda)(b-\lambda)\left(\alpha_{0}-\lambda\right)}}=-\frac{p}{4} .
$$

Последнее равенство получается интегрированием голоморфной 1-формы вдоль двух эквивалентных циклов на эллиптической кривой

$$
\mathscr{E}_{\alpha_{0}}: \mu^{2}=(a-\lambda)(b-\lambda)\left(\alpha_{0}-\lambda\right) .
$$

Мы уже упоминали в доказательстве теоремы 3.9 дифференциальные уравнения (4), задающие касательные к $\mathscr{C}_{\alpha_{0}}$ в эллиптических координатах. Мы видим, что $\sigma$ переводит эти касательные в прямые $x \pm y=$ const. Предложение доказано.

С помощью $\sigma$ биллиардная система в области, ограниченной несколькими софокусными кониками, трансформируется в биллиард в прямоугольном многоугольнике. По построению, поток в направлении $\pi / 4$ будет иметь те же траектории, что и исходный поток.

ПРимеР 3.14. Рассмотрим биллиард в области $\mathscr{D}_{0}$, граница которой состоит из двух половинок эллипсов и двух отрезков прямых, как на рис. 23. Будем счи-

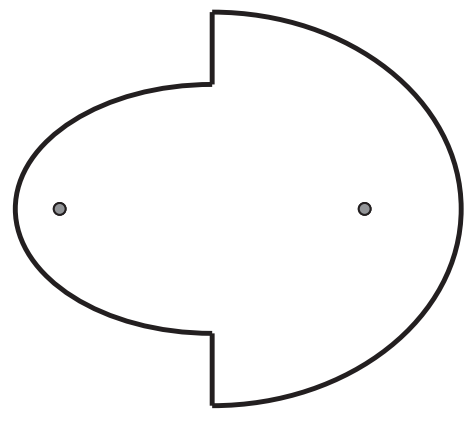

Рис. 23. Биллиардный стол $\mathscr{D}_{0}$

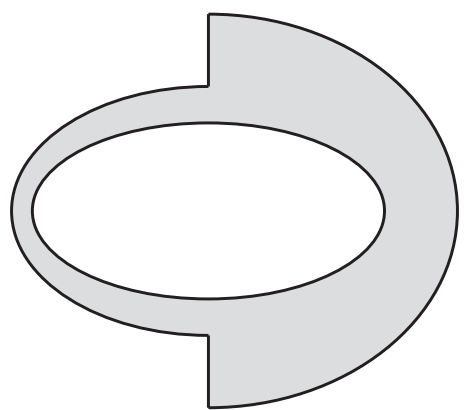

Рис. 24. Область $\mathscr{D}_{\alpha_{0}}$

тать, что каустика $\mathscr{C}_{\alpha_{0}}$ целиком лежит внутри области, и обозначим через $\mathscr{D}_{\alpha_{0}}$ пересечение $\mathscr{D}$ с областью, состоящей из касательных к $\mathscr{C}_{\alpha_{0}}$; см. рис. 24. Отображение $\sigma$ переводит $\mathscr{D}_{\alpha_{0}}$ в прямоугольный многоугольник, изображенный на рис. 25. Биллиардная система с каустикой $\mathscr{C}_{\alpha_{0}}$ в $\mathscr{D}_{0}$ орбитально эквивалентна биллиарду в прямоугольном многоугольнике $\sigma\left(\mathscr{D}_{\alpha_{0}}\right)$, у которого правая и левая стороны, обозначенные пунктиром, отождествляются параллельным сдвигом. 


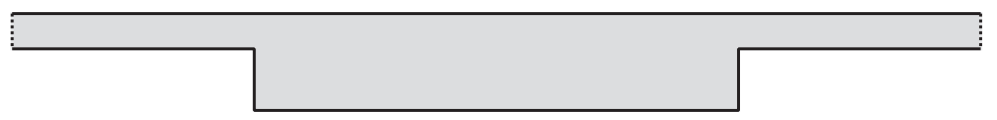

Рис. 25. Образ $\sigma\left(\mathscr{D}_{\alpha_{0}}\right)$

ПримеР 3.15. На рис. 26 представлен пример с островком. Если таких островков несколько, то $\sigma$ переведет биллиардный стол в область с рис. 27. Таким образом устанавливается связь псевдоинтегрируемой динамики с так называемой моделью “ветер-деревья" Эренфеста-Эренфест 1912, предложенной Паулем и Татьяной Эренфестами в 1912 г. [25]. Они использовали ее для исследований по статистической интерпретации второго закона термодинамики
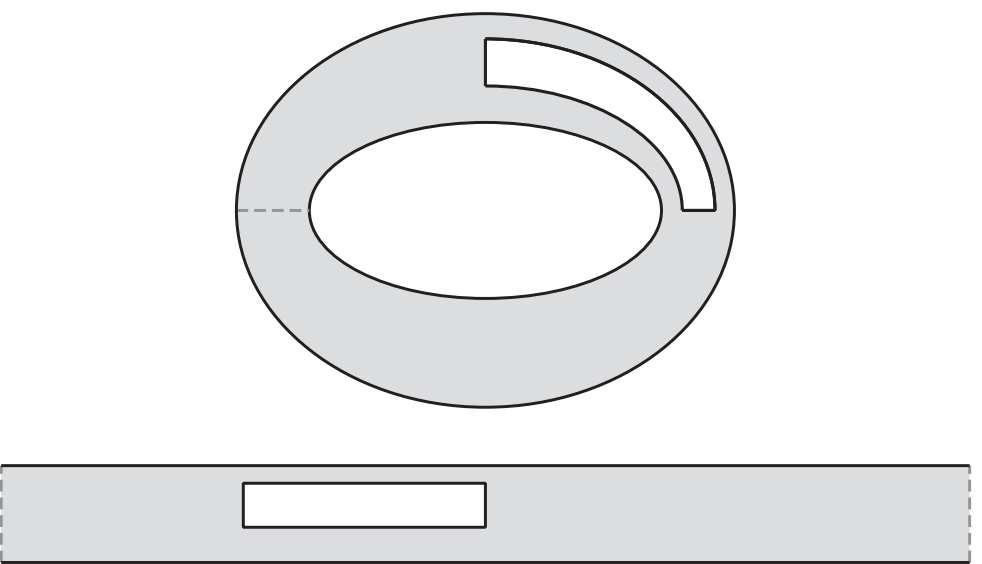

Рис. 26. Биллиард внутри эллипса с "островком", ограниченным дугами четырех софокусных квадрик, имеющий каустикой эллипс, и соответствующий ему прямоугольный многоугольник

и приложимости уравнения Больцмана. В их модели биллиардная частица сталкивается со случайным образом расставленными прямоугольными рассеивателями с параллельными сторонами; см. рис. 27. Подробнее с моделью “ветер-деревья" можно ознакомиться в [28], [44] и цитируемой там литературе.

Биллиардные столы с прямыми углами недавно, в другом контексте, были рассмотрены в [5]. Одно из важных отличий заключается в том, что в [5] рассматриваются все направления движения на заданном биллиардном столе, в то время как мы задаем направление, фиксируя каустику.

3.4. Арифметические и эргодические свойства. В этом пункте мы установим связь между биллиардами в областях, ограниченных несколькими софокусными кониками и преобразованиями перекладывания отрезков. Эта связь позволяет выявить два важных и интересных свойства - независимость динамических свойств псевдоинтегрируемых биллиардов от пучка софокусных квадрик (см. теорему 3.16) и существование биллиардных потоков, являющихся минимальными и не строго эргодическими (теорема 3.18 ). 


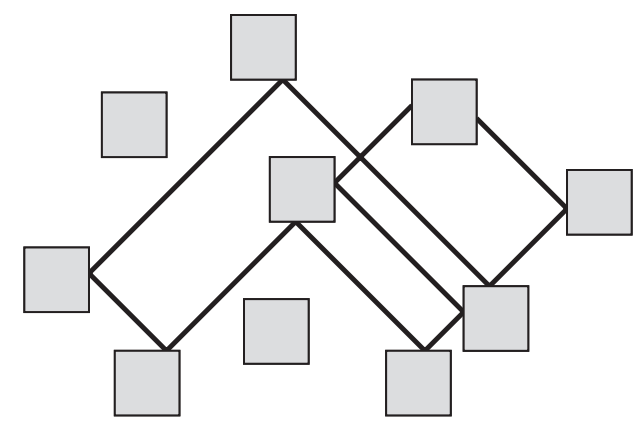

Рис. 27. Траектория в эренфестовской модели "ветер-деревья"

Теорема 3.16. Динамика биллиарда в области $\mathscr{D}_{0}$ (см. пример 3.14 и рис. 23) с эллипсом $\mathscr{C}_{\alpha_{0}}$ в качестве каустики не зависит от параметров $a, b$ софокусного семейства, а зависит только от чисел вращения $\rho\left(\lambda_{1}\right), \rho\left(\lambda_{2}\right)$. Здесь $\lambda_{1}$ и $\lambda_{2}$ - параметры эллипсов из семейства (1), задающих гранииу $\mathscr{D}_{0}$.

ДокАЗАТЕльство. Как мы видели в примере 3.14 , биллиардный поток с каустикой $\mathscr{C}_{\alpha_{0}}$ в $\mathscr{D}_{0}$ (см. пример 3.14 и рис. 23 ) эквивалентен биллиардному потоку в направлении $\pi / 4$ в прямоугольном многоугольнике на рис. 25 . Поскольку отношение сторон этого многоугольника можно выразить через числа вращения, утверждение доказано.

ПримеР 3.17. Рассмотрим траектории биллиарда с каустикой $\mathscr{C}_{\alpha_{0}}$ в области $\mathscr{D}_{0}$; см. пример 3.14 и рис. 23. Пусть числа вращения, соответствующие эллипсам $\mathscr{C}_{\lambda_{1}}$ и $\mathscr{C}_{\lambda_{2}}$, равны

$$
r_{1}=\frac{5}{11}+\frac{1}{22 \pi}, \quad r_{2}=\frac{5}{11}-\frac{1}{220 \pi} .
$$

Поскольку $r_{1}+10 r_{2}=5$, можно ожидать периодических биллиардных орбит. Биллиардный стол удовлетворяет условиям типа Кэли существования периодических биллиардных траекторий, выведенным в [14]. В этой статье были рассмотрены только биллиардные системы в областях, ограниченных несколькими софокусными квадриками, и условие типа Кэли было необходимо и достаточно для периодичности траекторий. Заметим, что эти условия действуют и в псевдоинтегрируемом смысле, но лишь как необходимые условия.

Теперь выпишем соответствующее преобразование перекладывания отрезков:

$$
\begin{gathered}
\Pi=\left(\begin{array}{cccccccc}
A & B & C & D & E & F & G & H \\
D & B & E & C & H & F & A & G
\end{array}\right) \\
\lambda=\left(\frac{1}{20 \pi}, \frac{1}{2}, \frac{1}{22}-\frac{21}{220 \pi}, \frac{5}{11}+\frac{1}{22 \pi}, \frac{1}{20 \pi}, \frac{1}{2}, \frac{1}{22}-\frac{21}{220 \pi}, \frac{5}{11}+\frac{1}{22 \pi}\right) .
\end{gathered}
$$

Можно проверить, что это минимальное преобразование.

Таким образом, хотя условие периодичности типа Кэли и выполнено, замкнутых траеторий не просто нет, но более того, каждая траектория плотна в кольце между краем биллиардного стола и каустикой. 
Теорема 3.18. Существуют биллиардные столь, ограниченные дугами нескольких софокусных коник и каустиками, в которых биллиардные потоки минимальны и не строго эргодичны.

ДокАзАТЕльство. Рассмотрим биллиардный стол на рис. 21 слева.

Соответствующий биллиардный поток эквивалентен дискретной динамике точек соприкосновения тректории с каустикой, учитывающей направление движения. Если ввести меру $\mu$ на каустике как в предложении 2.1 и параметризовать каустику в соответствии с $\mu$, то динамика переходит в следующее преобразование перекладывания отрезков на интервале $[-1,1)$ :

$$
\xi \mapsto \begin{cases}\xi+r_{1}, & \xi \in\left[-1,-r_{1}\right), \\ \xi+r_{1}-1, & \xi \in\left[-r_{1}, r_{2}-r_{1}\right), \\ \xi+r_{1}, & \xi \in\left[r_{2}-r_{1}, 1-r_{1}\right), \\ \xi+r_{1}-1, & \xi \in\left[1-r_{1}, r_{2}-r_{1}+1\right), \\ \xi+r_{1}-2, & \xi \in\left[r_{2}-r_{1}+1,1\right),\end{cases}
$$

где $r_{1}$ и $r_{2}$ - числа вращения внешнего эллипса и эллипса, проходящего через вершину невыпуклого угла относительно каустики.

Преобразование (7) отвечает примеру Вича минимальной, но не строго эргодической системы [45], см. также [38]. А именно, пусть $r_{1}$ - иррациональное число с неограниченными значениями подходящих дробей. Тогда существуют такие иррациональные $r$, что при $r_{2}=r_{1}-r$ мера $\mu$ на каустике не эргодическая и, значит, не строго эргодическая.

ЗАмечАниЕ 3.19. Используя технику, аналогичную [38], можно показать, что существует бесконечно много биллиардных столов, ограниченных дугами софокусных коник, в которых соответствующие биллиардные потоки минимальны и не являются строго эргодическими. Соответствующие преобразования перекладывания, вообще говоря, отличаются от преобразований в примере Вича.

\section{4. Интегрируемые конгруэнции прямых и решетки двойных отражений}

Дискретная дифференциальная геометрия как раздел современной науки зародилась совсем недавно (см. [8]), при изучении геометрии решеток. Фундаментальную роль в ней играют так называемые условия интегрируемости для квад-графов. С другой стороны, одной из важнейших и интереснейших страниц дифференциальной геометрии являются геодезические на эллипсоиде. Как известно, биллиардные системы внутри квадрик являются естественной дискретизацией систем геодезических на эллипсоидах. Ниже мы представим их как кирпичики из числа лежащих в основании дискретной дифференциальной геометрии. 
Основными элементами систем на квад-графах являются уравнения вида $Q\left(x, x_{1}, x_{2}, x_{12}\right)=0$ на четырехугольниках, где $Q$ - мультиаффинный полином, имеющий степень 1 по каждому переменному. Такие уравнения называются квад-уравнениями. Полевые переменные $x_{i}$ сопоставляются вершинам четырехугольника, как на рис. 28. Мы можем решить квад-уравнение по каждой

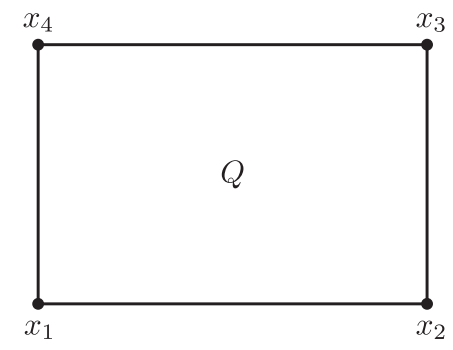

Рис. 28. Квад-уравнение $Q\left(x_{1}, x_{2}, x_{3}, x_{4}\right)=0$

переменной, и решение оказывается рациональной функцией остальных трех переменных. Вслед за [2] будем трактовать интегрируемость как совместность; см. рис. 29. Сопоставим граням куба шесть квад-уравнений и будем говорить,

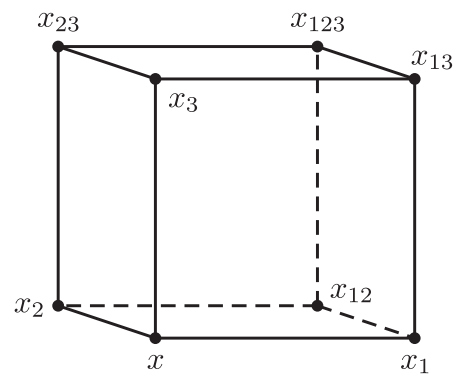

Рис. 29. 3D-совместность

что система $3 \mathrm{D}$-совместна, если три значения $x_{123}$, полученные решением уравнений с правой, задней и верхней граней, совпадают для любых начальных данных $\left\{x, x_{1}, x_{2}, x_{3}\right\}$.

Здесь нас будет интересовать геометрический вариант интегрируемых квадграфов, в котором прямые играют роль вершинных полей.

Мы посвятим п. 4.1 интерпретации в терминах квад-графов некоторых результатов из [16], полученных с использованием биллиардной алгебры. В п. 4.2 мы вернемся к понятию решетки двойных отражений, введенному в [20], и разовьем его; см. определение 4.3 и замечание 4.4. В теореме 4.5 мы покажем, как строить решетки из этого расширенного класса, исходя из нескольких биллиардных траекторий в областях, ограниченных софокусными квадриками, а затем приведем несколько новых примеров. В п. 4.3 мы построим отображение Янга-Бакстера, связанное с семейством софокусных квадрик. 
4.1. Биллиардная алгебра и квад-графы. Начнем с теоремы о семействах софокусных квадрик из [16].

ТЕОрема 4.1 (теорема о шестиконечной звезде). Пусть $\mathscr{F}$ - семейство софокусных квадрик в трехмерном пространстве. Тогда в пространстве есть конфигурация из 12 плоскостей со следующими свойствами.

(i) Плоскости можно сгруппировать в 8 триплетов так, что плоскости в каждом триплете касаются некоторой квадрики из Ғ (своей для каждого триплета) и три точки касания коллинеарны. Каждая плоскость из конфигурачии попадает в два триплета.

(ii) Плоскости можно сгруппировать в 6 четверок так, что плоскости в каждой четверке входят в один пучок плоскостей и касаются двух различных квадрик из $\mathscr{F}$. Каждая плоскость из конфигурачии попадает в две четверки.

Кроме того, такая конфигурачия задается тремя плоскостями, касающимися трех различных квадрик из Ғ с коллинеарными точками касания.

Мы приводим такую конфигурацию плоскостей из двойственного пространства на рис 30: каждая плоскость соответствует вершине ломаной.

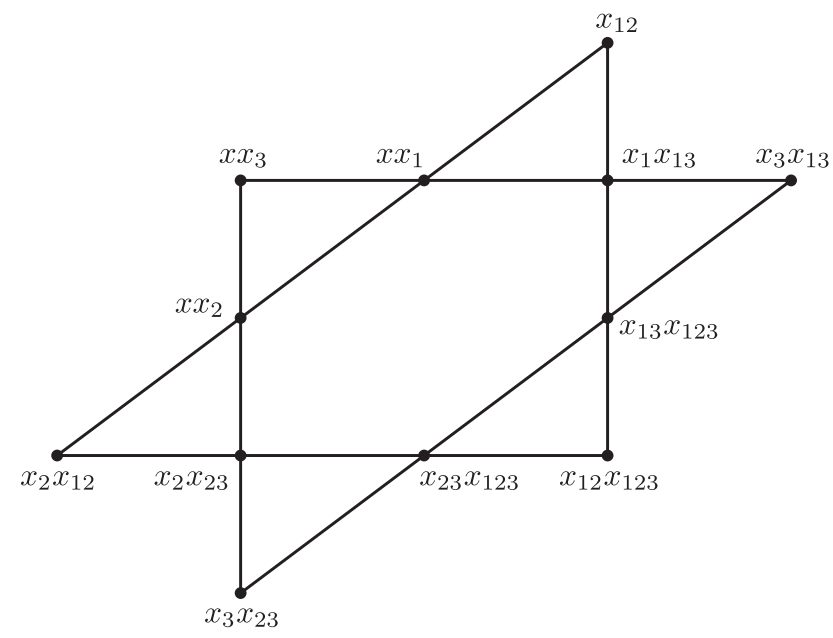

Рис. 30. Конфигурация плоскостей из теоремы 4.1

Для разъяснения обозначений, используемых на рис. 30, напомним конструкцию, приводящую к конфигурации из теоремы 4.1. Пусть $\mathscr{Q}_{1}, \mathscr{Q}_{2}, \mathscr{Q}_{3}-$ квадрики из $\mathscr{F}$, а $\alpha, \beta, \gamma-$ соответствующие касательные плоскости, причем точки касания $A, B$ и $C$ коллинеарны. Через $x$ обозначим прямую, содержащую эти точки, а через $x_{1}, x_{2}, x_{3}$ - прямые, полученные из $x$ отражениями от $\mathscr{Q}_{1}, \mathscr{Q}_{2}, \mathscr{Q}_{3}$ в точках $A, B, C$ соответственно.

Теперь аналогично предложению 2.8 найдем прямые $x_{12}, x_{13}, x_{23}, x_{123}$, дополняющие триплеты $\left\{x, x_{1}, x_{2}\right\},\left\{x, x_{1}, x_{3}\right\},\left\{x, x_{2}, x_{3}\right\},\left\{x_{3}, x_{13}, x_{23}\right\}$ до конфигураций двойного отражения.

На рис. 30 выделим следующие объекты. 
12 вершин. Каждой вершине сопоставлены касательная плоскость к одной из квадрик $\mathscr{Q}_{1}, \mathscr{Q}_{2}, \mathscr{Q}_{3}$ и пара прямых, которые переводятся одна в другую отражением от квадрики в точке касания с упомянутой плоскостью.

8 треугольников. Для каждого треугольника плоскости, сопоставленные его вершинам, касаются соответствующих квадрик в трех коллинеарных точках - так что всякому треугольнику естественным образом сопоставляется прямая, проходящая через эти точки.

6 ребер. На каждом ребре лежат четыре вершины, и четыре плоскости, им сопоставленные, принадлежат к одному пучку плоскостей; так что каждому ребру соответствует конфигурация двойного отражения.

Теперь мы можем доказать 3D-совместность квад-уравнений, задаваемых с помощью конфигураций двойных отражений. Смысл следующей теоремы заключается в том, что отражения от трех квадрик коммутируют.

ТЕОРема 4.2. Пусть $x, x_{1}, x_{2}, x_{3}$ - прямые в проективном пространстве, причем $x_{1}, x_{2}, x_{3}$ получаются из $x$ отражсением от софокусных квадрик $\mathscr{Q}_{1}$, $\mathscr{Q}_{2}, \mathscr{Q}_{3}$ соответственно. Пусть $x_{12}, x_{13}, x_{23}, x_{123}$ - такие прямые, что следующие четверки являются конфигурачиями двойного отражения:

$$
\left\{x, x_{1}, x_{12}, x_{2}\right\}, \quad\left\{x, x_{1}, x_{13}, x_{3}\right\}, \quad\left\{x, x_{2}, x_{23}, x_{3}\right\}, \quad\left\{x_{1}, x_{12}, x_{123}, x_{13}\right\} .
$$

Тогда следующие четверки также являются конфигуращиями двойного отражения:

$$
\left\{x_{2}, x_{12}, x_{123}, x_{23}\right\}, \quad\left\{x_{3}, x_{13}, x_{123}, x_{23}\right\} .
$$

ДокАЗАтеЛьство. Заметим, что конфигурация из теоремы 4.1, очевидно, имеет комбинаторную структуру куба, если плоскостям сопоставить ребра куба. Тогда прямые $x, x_{1}, x_{2}, x_{3}, x_{12}, x_{13}, x_{23}, x_{123}$ будут соответствовать вершинам куба как показано на рис. 29. Пара прямых соответствует концам ребра куба, если они переводятся друг в друга отражением от плоскости, сопоставленной этому ребру. Грани куба соответствуют конфигурациям двойного отражения. Заметим, кроме того, что плоскости, сопоставленные параллельным ребрам куба, касаются одной и той же квадрики. Это следует из теоремы 4.1 и построения, следующего за этой теоремой; см. рис. 30.

4.2. Решетки двойных отражений. Зафиксируем $d-1$ квадрик из семейства софокусных квадрик (2), и пусть $A \subset \mathscr{L}^{d}-$ множество прямых, касающихся этих $d-1$ квадрик.

ОПРЕДЕЛЕниЕ 4.3. Решетка двойных отражений - это отображение

$$
\varphi: \mathbb{Z}^{m} \rightarrow \mathscr{A}
$$

для которого прямые $\varphi\left(\mathbf{n}_{0}\right), \varphi\left(\mathbf{n}_{0}+\mathbf{e}_{i}\right), \varphi\left(\mathbf{n}_{0}+\mathbf{e}_{j}\right), \varphi\left(\mathbf{n}_{0}+\mathbf{e}_{i}+\mathbf{e}_{j}\right)$ образуют конфигурацию двойного отражения для любых $i, j \in\{1, \ldots, m\}, i \neq j$, и $\mathbf{n}_{0} \in \mathbb{Z}^{m}$.

Если вдобавок для всякого $j \in\{1, \ldots, m\}$ и $\mathbf{n}_{0} \in \mathbb{Z}^{m}$ последовательность $\left\{\varphi\left(\mathbf{n}_{0}+i \mathbf{e}_{j}\right)\right\}_{i \in \mathbb{Z}}$ задает биллиардную траекторию внутри квадрики из семейства (2), то будем называть $\varphi$ биллиардной решеткой двойнъх отражений. 
ЗАмЕчАНИЕ 4.4. Заметим, что биллиардные решетки двойных отражений были введены авторами в [20], где мы назвали их просто решетками двойных отражений.

Следующее утверждение объясняет, как построить решетку двойных отражений, если она уже задана на осях координат.

Теорема 4.5. Пусть $\left(\ell_{i}^{j}\right)_{i \in \mathbb{Z}}, 1 \leqslant j \leqslant m$, суть $m$ последовательностей из $\mathscr{A}$ со следующими свойствами: любые две соседние прямые из последовательности компланарны и $\ell_{0}^{1}=\cdots=\ell_{0}^{m}$.

Тогда существует единственная решетка двойных отражений $\varphi: \mathbb{Z}^{m} \rightarrow \mathscr{A}$, для которой $\varphi\left(n \mathbf{e}_{j}\right)=\ell_{n}^{j}, n \in \mathbb{Z}$.

ДокАЗАТЕЛЬСтво. $\varphi$ можно продолжить на всё $\mathbb{Z}^{m}$, потребовав, чтобы прямые, сопоставленные вершинам единичного квадрата решетки, образовывали конфигурацию двойного отображения.

Корректность этой конструкции следует из теоремы 4.2.

В следующем примере 4.6, используя обобщение большой теоремы Понселе, мы строим конечную решетку двойных отражений.

ПримеР 4.6. Пусть $\mathscr{Q}_{\alpha_{1}}, \ldots, \mathscr{Q}_{\alpha_{m}}$ - квадрики из $(2)$, для которых имеется биллиардная траектория с периодом $m$, последовательно отражающаяся от $\mathscr{Q}_{\alpha_{1}}, \ldots, \mathscr{Q}_{\alpha_{m}}$. В качестве $\mathscr{A}$ возьмем множество прямых в пространстве с тем же набором $d-1$ каустик, что и данная периодическая траектория. Тогда по обобщенной большой теореме Понселе существует периодическая траектория, начинающаяся с любой прямой из $\mathscr{A}$; более того, порядок отражений произволен [10].

Зафиксируем прямую $\ell_{0} \in \mathscr{A}$. Тогда для всякой перестановки $\left(p_{1}, \ldots, p_{m}\right)$ множества $\{1, \ldots, m\}$ существует периодическая биллиардная траектория, начинающаяся с $\ell_{0}$ и последовательно отражающаяся от $\mathscr{Q}_{\alpha_{p_{1}}}, \ldots, \mathscr{Q}_{\alpha_{p_{m}}}$. На базе таких траекторий с помощью теоремы 4.5 мы строим решетку двойных отражений $\varphi_{0}: \mathbb{Z}^{m !} \rightarrow \mathscr{A}$.

Говорят, что две решетки двойных отражений $\varphi_{0}, \varphi_{1}$ связаны $F$-преобразованием, если для всякого $\mathbf{n}$ прямые $\varphi_{0}(\mathbf{n})$ и $\varphi_{1}(\mathbf{n})$ пересекаются [8]. Следующий пример 4.7 показывает, что две решетки двойных отражений из примера 4.6 можно связать конечной последовательностью $F$-преобразований.

ПримеР 4.7. В условиях примера 4.6 возьмем еще одну прямую $\ell_{1} \in \mathscr{A}$ и построим соответствующую решетку двойных отражений $\varphi_{1}$. Поскольку $\ell_{1}$ можно получить из $\ell_{0}$ не более чем $d-1$ отражением от квадрик из семейства (2) [16], [18], существует отображение $\Phi: \mathbb{Z}^{m !} \times\{0, \ldots, d-1\} \rightarrow \mathscr{A}$ такое, что

$$
\left.\Phi\right|_{\mathbb{Z}^{m ! \times\{0\}}}=\varphi_{0},\left.\quad \Phi\right|_{\mathbb{Z}^{m ! \times\{d-1\}}}=\varphi_{1} .
$$

Оно определено однозначно и может быть построено как объясняется в теореме 4.5. Две последовательные решетки двойных отражений $\left.\Phi\right|_{\mathbb{Z}^{m !} \times\{i-1\}}$ и $\left.\Phi\right|_{\mathbb{Z}^{m !} \times\{i\}}$ связаны $F$-преобразованием, поскольку соответствующие прямые из этих сеток пересекаются. 
В примере 4.8 мы строим решетку двойных отражений, связанную с псевдоинтегрируемыми биллиардами.

ПримеР 4.8. Рассмотрим биллиардные столы $\mathscr{D}^{\prime}$ и $\mathscr{D}^{\prime \prime}$, ограниченные дугами софокусных коник из семейства (1). Будем считать также, что семейство (1) удовлетворяет условию $a=b$, так что оно состоит из концентрических окружностей и прямых, проходящих через их общий центр. Рассмотрим биллиардные траектории на каждом из столов, начинающиеся с фиксированной прямой $\ell_{0}$ на плоскости. По теореме 4.5 они приводят к решетке двойных отражений $\varphi: \mathbb{Z}^{2} \rightarrow \mathscr{A}$, где $\mathscr{A}-$ множество прямых на плоскости, касающихся каких-то из концентрических окружностей и $\ell_{0} \in \mathscr{A}$. Вдобавок, последовательности $\left(\varphi\left(\mathbf{n}+i \mathbf{e}_{1}\right)\right)_{i \in \mathbb{Z}},\left(\varphi\left(\mathbf{n}+i \mathbf{e}_{2}\right)\right)_{i \in \mathbb{Z}}$ соответствуют биллиардным траекториям, получающимся из $\mathscr{D}^{\prime}$ и $\mathscr{D}^{\prime \prime}$ симметриями софокусного семейства, т. е. изометрическими преобразованиями, сохраняющими центр.

В последнюю пару лет динамика биллиардов в областях, ограниченных пучками квадрик, также рассматривалась в пседоевклидовой постановке; см. [19], [32], а также [21]. Несколько примеров сеток двойных отражений в псевдоевклидовых пространствах построено в [20], [23]. Более свежие конструкции биллиардов, связанные с конфигурациями двойного отражения в псевдоевклидовых пространствах, можно найти в [31].

4.3. Отображения Янга-Бакстера. Отображением Янга-Бакстера называется отображение $R: \mathscr{X} \times \mathscr{X} \rightarrow \mathscr{X} \times \mathscr{X}$, удовлетворяющее уравнению Янга-Бакстера

$$
R_{23} \circ R_{13} \circ R_{12}=R_{12} \circ R_{13} \circ R_{23},
$$

где $R_{i j}: \mathscr{X} \times \mathscr{X} \times \mathscr{X} \rightarrow \mathscr{X} \times \mathscr{X} \times \mathscr{X}$ действует как $R$ на $i$-й и $j$-й сомножители произведения и тождественным образом на оставшийся сомножитель; см. [1] и ссылки там.

Здесь мы построим пример отображения Янга-Бакстера, связанного с семейством софокусных квадрик. Сначала зафиксируем семейство софокусных квадрик из $\mathbf{C P}^{n}$ :

$$
\mathscr{Q}_{\lambda}: \frac{z_{1}^{2}}{a_{1}-\lambda}+\cdots+\frac{z_{d}^{2}}{a_{d}-\lambda}=z_{n+1}^{2},
$$

где $a_{1}, \ldots, a_{d}$ - скаляры из $\mathbb{C}$, a $\left[z_{1}: z_{2}: \cdots: z_{n+1}\right]$ - однородные координаты в $\mathbf{C P}^{n}$.

Пусть $\mathscr{X}$ - двойственное пространство $\mathbf{C P}^{n *} n$-мерного проективного пространства, т. е. многообразие гиперплоскостей из $\mathbf{C P}^{n}$. Заметим, что общая гиперплоскость касается ровно одной квадрики из (8). Вдобавок, в общем пучке гиперплоскостей ровно две плоскости касаются фиксированной общей квадрики.

Теперь рассмотрим пару гиперплоскостей $x, y$. Они касаются единственных квадрик $\mathscr{Q}_{\alpha}$ и $\mathscr{Q}_{\beta}$ из (8) соответственно. Вдобавок, они задают пучок гиперплоскостей. В этом пучке есть еще ровно две гиперплоскости $x^{\prime}$ и $y^{\prime}$, отличные от $x$ и $y$ и касающиеся $\mathscr{Q}_{\alpha}$ и $\mathscr{Q}_{\beta}$ соответственно, как изображено на рис. 31. 


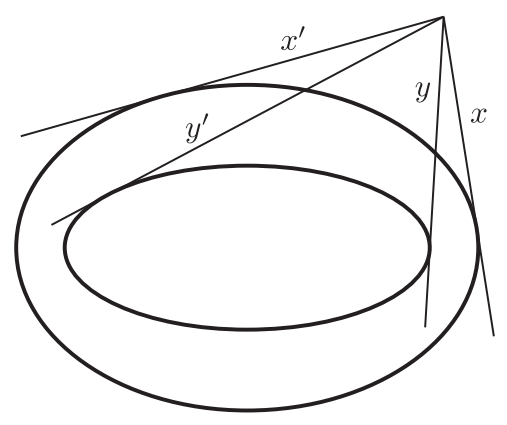

Рис. 31. $R(x, y)=\left(x^{\prime}, y^{\prime}\right)$

Зададим $R: \mathbf{C P}^{n *} \times \mathbf{C P}^{n *} \rightarrow \mathbf{C P}^{n *} \times \mathbf{C P}^{n *}$ так, чтобы $R(x, y)=\left(x^{\prime}, y^{\prime}\right)$, если $\left(x^{\prime}, y^{\prime}\right)$ получается из $(x, y)$ описанным выше способом; см. рис. 31.

Тогда отображения

$$
R_{12}, R_{13}, R_{23}: \mathbf{C P}^{n *} \times \mathbf{C P}^{n *} \times \mathbf{C P}^{n *} \rightarrow \mathbf{C P}^{n *} \times \mathbf{C P}^{n *} \times \mathbf{C P}^{n *}
$$

задаются следующим образом:

$$
\begin{array}{ll}
R_{12}(x, y, z)=\left(x^{\prime}, y^{\prime}, z\right), & \text { где }\left(x^{\prime}, y^{\prime}\right)=R(x, y) ; \\
R_{13}(x, y, z)=\left(x^{\prime}, y, z^{\prime}\right), & \text { где }\left(x^{\prime}, z^{\prime}\right)=R(x, z) ; \\
R_{23}(x, y, z)=\left(x, y^{\prime}, z^{\prime}\right), & \text { где }\left(y^{\prime}, z^{\prime}\right)=R(y, z) .
\end{array}
$$

Чтобы проверить уравнения Янга-Бакстера для $R$, нам нужна следующая лемма.

Лемма 4.9. Пусть $\mathscr{Q}_{\alpha}, \mathscr{Q}_{\beta}, \mathscr{Q}_{\gamma}-$ три невырожденные квадрики из семейства (8), а х, y, z-касательные плоскости к ним. Положим

$$
\left(x_{2}, y_{1}\right)=R(x, y), \quad\left(x_{3}, z_{1}\right)=R(x, z), \quad\left(y_{3}, z_{2}\right)=R(y, z) .
$$

Пусть $x_{23}, y_{13}, z_{12}$ - общие гиперплоскости пучков гиперплоскостей, заданньх парами $\left(x_{3}, y_{3}\right)$ u $\left(x_{2}, z_{2}\right),\left(x_{3}, y_{3}\right) u\left(y_{1}, z_{1}\right),\left(y_{1}, z_{1}\right) u\left(x_{2}, z_{2}\right)$ соответственно.

Тогда $x_{23}, y_{13}$ и $z_{12}$ касаются $\mathscr{Q}_{\alpha}, \mathscr{Q}_{\beta}$ и $\mathscr{Q}_{\gamma}$ соответственно.

ДокАЗАтельство. Это утверждение в размерности $n=2$, сформулированное в терминах двойственного пространства, было доказано в [1; теорема 5].

В произвольной размерности $n$ рассмотрим двойственную ситуацию. Двойственные квадрики $\mathscr{Q}_{\alpha}^{*}, \mathscr{Q}_{\beta}^{*}$ и $\mathscr{Q}_{\gamma}^{*}$ принадлежат одному линейному пучку, а точки $x^{*}, y^{*}$ и $z^{*}$, двойственные гиперплоскостям $x, y$ и $z$, лежат на этих квадриках соответственно. Рассмотрим двумерную плоскость, содержащую эти три точки. Пересечение пучка квадрик с этой плоскостью (как и с любой другой) задает пучок коник. Таким образом, теорема 5 из [1] выполнена во всех размерностях.

Наконец, рассматриваемая лемма двойственна к этой теореме, что и завершает доказательство. 
Теорема 4.10. Отображение $R$ удовлетворяет уравнению Янга-Бакстера.

ДокАЗАТЕЛЬСтво. Пусть $x, y, z$ - гиперплоскости в $\mathbf{C P}^{n}$. Мы хотим доказать, что

$$
R_{23} \circ R_{13} \circ R_{12}(x, y, z)=R_{12} \circ R_{13} \circ R_{23}(x, y, z) .
$$

Пусть $\mathscr{Q}_{\alpha}, \mathscr{Q}_{\beta}, \mathscr{Q}_{\gamma}$ - квадрики из семейства (8), касающиеся $x, y$ и $z$ соответственно.

Запишем

$$
\begin{aligned}
& (x, y, z) \stackrel{R_{12}}{\longrightarrow}\left(x_{2}, y_{1}, z\right) \stackrel{R_{13}}{\longrightarrow}\left(x_{23}, y_{1}, z_{1}\right) \stackrel{R_{23}}{\longrightarrow}\left(x_{23}, y_{13}, z_{12}\right), \\
& (x, y, z) \stackrel{R_{23}}{\longrightarrow}\left(x, y_{3}, z_{2}\right) \stackrel{R_{13}}{\longrightarrow}\left(x_{3}, y_{3}, z_{12}^{\prime}\right) \stackrel{R_{12}}{\longrightarrow}\left(x_{23}^{\prime}, y_{13}^{\prime}, z_{12}^{\prime}\right) .
\end{aligned}
$$

Теперь применим лемму 4.9 к гиперплоскостям $x, y, z_{2}$. Поскольку

$$
\left(x_{2}, y_{1}\right)=R(x, y), \quad\left(x_{3}, z_{12}^{\prime}\right)=R\left(x, z_{2}\right), \quad\left(y_{3}, z\right)=R\left(y, z_{2}\right),
$$

мы видим, что общая гиперплоскость пучков, содержащих $\left(x_{3}, y_{3}\right)$ и $\left(x_{2}, z\right)$, касается $\mathscr{Q}_{\alpha}$ и, значит, совпадает с $x_{23}$ и $x_{23}^{\prime}$, т. е. $x_{23}=x_{23}^{\prime}$. Далее, общая гиперплоскость пучков, содержащих $\left(y_{1}, z_{12}^{\prime}\right)$ и $\left(x_{2}, z\right)$, касается $\mathscr{Q}_{\gamma}$ и, значит, совпадает с $z_{1}$ и с $z_{12}=z_{12}^{\prime}$. Наконец, общая гиперплоскость пучков, содержащих $\left(x_{3}, y_{3}\right)$ и $\left(y_{1}, z_{12}^{\prime}\right)$, касается $\mathscr{Q}_{\beta}$ и, значит, совпадает с $y_{13}=y_{13}^{\prime}$, что и завершает доказательство.

ЗАмечАНИЕ 4.11. Вместо того чтобы задавать $R$ на всем пространстве $\mathbf{C P}^{n *} \times \mathbf{C P}^{n *}$, мы можем рассмотреть его ограничение на произведение двух невырожденных квадрик из (8):

$$
R(\alpha, \beta): \mathscr{Q}_{\alpha}^{*} \times \mathscr{Q}_{\beta}^{*} \rightarrow \mathscr{Q}_{\alpha}^{*} \times \mathscr{Q}_{\beta}^{*},
$$

где пара $(x, y)$ касательных гиперплоскостей отображается в такую пару $\left(x_{1}, y_{1}\right)$, что $x, y, x_{1}, y_{1}$ принадлежат одному пучку.

Соответствующее уравнения Янга-Бакстера имеет вид

$$
R_{23}(\beta, \gamma) \circ R_{13}(\alpha, \gamma) \circ R_{12}(\alpha, \beta)=R_{12}(\alpha, \beta) \circ R_{13}(\alpha, \gamma) \circ R_{23}(\alpha, \beta),
$$

где с обеих сторон стоят отображения из $\mathscr{Q}_{\alpha}^{*} \times \mathscr{Q}_{\beta}^{*} \times \mathscr{Q}_{\gamma}^{*}$ в себя.

В [1] для неприводимых алгебраических многообразий $\mathscr{X}_{1}$ и $\mathscr{X}_{2}$ было определено квадрирачионалъное отображсние $F: \mathscr{X}_{1} \times \mathscr{X}_{2}$. Для такого отображения $F$ и произвольной пары $(x, y) \in \mathscr{X}_{1} \times \mathscr{X}_{2}$, за исключением нескольких замкнутых подмногообразий коразмерности не меньше 1 , график $\Gamma_{F} \subset$ $\mathscr{X}_{1} \times \mathscr{X}_{2} \times \mathscr{X}_{1} \times \mathscr{X}_{2}$ пересекает каждое из множеств $\{x\} \times\{y\} \times \mathscr{X}_{1} \times \mathscr{X}_{2}$, $\mathscr{X}_{1} \times \mathscr{X}_{2} \times\{x\} \times\{y\}, \mathscr{X}_{1} \times\{y\} \times\{x\} \times \mathscr{X}_{2},\{x\} \times \mathscr{X}_{2} \times \mathscr{X}_{1} \times\{y\}$ в единственной точке (см. [1; определение 3$])$. Иными словами, $\Gamma_{F}$ является графиком четырех отображений: $F, F^{-1}, \bar{F}$ и $\bar{F}^{-1}$.

Следующее утверждение обобщает предложение 4 из [1].

ПреДЛОЖЕНИЕ 4.12. Отображение $R(\alpha, \beta): \mathscr{Q}_{\alpha}^{*} \times \mathscr{Q}_{\beta}^{*} \rightarrow \mathscr{Q}_{\alpha}^{*} \times \mathscr{Q}_{\beta}^{*} \kappa в а д р и$ рациональное. Оно является инволюиией и совпадает с отображением-компаньоном $\bar{R}(\alpha, \beta)$. 


\section{5. Обсуждение}

Важно подчеркнуть, что обычно при изучении биллиардов в многоугольниках рассматриваются все направления на заданном биллиардном столе, в то время как здесь мы предписываем направление фиксацией каустики. Встает естественный вопрос об анализе поведения нашей конструкции, когда край биллиардного стола зафиксирован, а каустика меняется, так что возникает кривая в пространстве Гурвица комплексных структур на эллиптических кривых (см. [26]). Выяснить, как описанная нами конструкция будет меняться вдоль такой кривой - интересный и заманчивый вопрос, которым мы рассчитываем заняться в дальнейшем.

С другой стороны, решетки двойных отражений открывают перспективы дальнейшего изучения связей между биллиардами в областях, ограниченных софокусными квадриками, и дискретными структурами.

Авторам посчастливилось в течение долгих лет поддерживать плодотворные товарищеские отношения с академиком В.В. Козловым и его школой. Они пользуются представившейся возможностью пожелать Валерию Васильевичу здоровья и дальнейших творческих успехов.

\section{Список литературы}

[1] V.E. Adler, A.I. Bobenko, Yu. B. Suris, "Geometry of Yang-Baxter maps: pencils of conics and quadrirational mappings", Comm. Anal. Geom., 12:5 (2004), 967-1007.

[2] V.E. Adler, A. I. Bobenko, Y. B. Suris, "Integrable discrete nets in Grassmannians", Lett. Math. Phys., 89:2 (2009), 131-139.

[3] В.И. Арнольд, Математические методы классической механики, Наука, М., 1974, 431 с.; англ. пер.: V. I. Arnold, Mathematical methods of classical mechanics, Grad. Texts in Math., 60, Springer-Verlag, New York, 1978, xvi+462 pp.

[4] В. И. Арнольд, "Полиинтегрируемые потоки", Алгебра и анализ, 4:6 (1992), 54-62; англ. пер.: V. I. Arnol'd, "Poly-integrable flows", St. Petersburg Math. J., 4:6 (1993), 1103-1110.

[5] J.S. Athreya, A. Eskin, A. Zorich, "Right-angled billiards and volumes of moduli spaces of quadratic differentials on $\mathbb{C} P^{1 "}, 2012$ (v3 - 2013), 61 pp., arXiv: 1212.1660.

[6] M. Berger, Geometry. I, Universitext, Springer-Verlag, Berlin, 1987, xiv+428 pp.

[7] M. Berger, Geometry. II, Universitext, Springer-Verlag, Berlin, 1987, x+406 pp.

[8] A. I. Bobenko, Y. B. Suris, Discrete differential geometry. Integrable structure, Grad. Stud. Math., 98, Amer. Math. Soc., Providence, RI, 2008, xxiv+404 pp.

[9] P. S. Casas, R. Ramírez-Ros, "The frequency map for billiards inside ellipsoids", SIAM J. Appl. Dyn. Syst., 10:1 (2011), 278-324.

[10] S.-J. Chang, B. Crespi, K.-J. Shi, "Elliptical billiard systems and the full Poncelet's theorem in $n$ dimensions", J. Math. Phys., 34:6 (1993), 2242-2256.

[11] M. Chasles, "Géométrie pure. Théorèmes sur les sections coniques confocales", Ann. Math. Pures Appl. [Ann. Gergonne], 18 (1827/1828), 269-276.

[12] G. Darboux, "Sur les polygones inscrits et circonscrits à l'ellipsoïde", Bulletin de la Société philomathique, 7 (1870), 92-94.

[13] G. Darboux, Leçons sur la théorie générale des surfaces et les applications géométriques du calcul infinitésimal, v. 2, 2-ème éd., Gauthier-Villars, Paris, 1915, xix+567 pp.; v. 3, 1-ère éd., 1894, xvi+501 pp.

[14] V. Dragović, M. Radnović, "Cayley-type conditions for billiards within $k$ quadrics in $\mathbb{R}^{d ",}$ J. Phys. A, 37:4 (2004), 1269-1276. 
[15] V. Dragović, M. Radnović, "Geometry of integrable billiards and pencils of quadrics", J. Math. Pures Appl. (9), 85:6 (2006), 758-790.

[16] V. Dragović, M. Radnović, "Hyperelliptic Jacobians as billiard algebra of pencils of quadrics: beyond Poncelet porisms", Adv. Math., 219:5 (2008), 1577-1607.

[17] В. Драгович, М. Раднович, "Интегрируемые биллиарды и квадрики", УМН, 65:2(392) (2010), 133-194; англ. пер.: V. Dragović, M. Radnović, "Integrable billiards and quadrics", Russian Math. Surveys, 65:2 (2010), 319-379.

[18] V. Dragović, M. Radnović, Poncelet porisms and beyond. Integrable billiards, hyperelliptic Jacobians and pencils of quadrics, Front. Math., Birkhäuser/Springer Basel AG, Basel, 2011, viii+293 pp.

[19] V. Dragović, M. Radnović, "Ellipsoidal billiards in pseudo-Euclidean spaces and relativistic quadrics", Adv. Math., 231:3-4 (2012), 1173-1201.

[20] V. Dragović, M. Radnović, "Billiard algebra, integrable line congruences, and double reflection nets", J. Nonlinear Math. Phys., 19:3 (2012), 1250019, 18 pp.

[21] V. Dragović, M. Radnović, "Minkowski plane, confocal conics, and billiards", Publ. Inst. Math. (Beograd) (N. S.), 94:108 (2013), 17-30.

[22] V. Dragović, M. Radnović, "Pseudo-integrable billiards and arithmetic dynamics", J. Mod. Dyn., 8:1 (2014), 109-132.

[23] V. Dragović, M. Radnović, "Bicentennial of the great Poncelet theorem (1813-2013): current advances", Bull. Amer. Math. Soc. (N. S.), 51:3 (2014), 373-445.

[24] V. Dragović, M. Radnović, "Periods of pseudo-integrable billiards", DOI: 10.1007/s40598-014-0004-0, Arnold Math. J., 2015 (to appear).

[25] P. Ehrenfest, T. Ehrenfest, "Begriffliche Grundlagen der statistischen Auffassung in der Mechanik", Encykl. Math. Wiss., IV, 2, II, B. G. Teubner, Leipzig, 1912, 90 pp.; англ. пер.: P. Ehrenfest, T. Ehrenfest, The conceptual foundations of the statistical approach in mechanics, Dover Publications, Inc., New York, 1990, xiv+114 pp.

[26] J. Fay, Kernel functions, analytic torsion, and moduli spaces, Mem. Amer. Math. Soc., 96, № 464, Amer. Math. Soc., Providence, RI, 1992, vi+123 pp.

[27] R. Hartshorne, Foundations of projective geometry, Lecture notes, Harvard University, 1966/1967, New York, 1967, vii+167 pp.

[28] P. Hubert, S. Lelièvre, S. Troubetzkoy, "The Ehrenfest wind-tree model: periodic directions, recurrence, diffusion", J. Reine Angew. Math., 656 (2011), 223-244.

[29] C. G. J. Jacobi, Fundamenta nova theoriae functiorum ellipticarum, Sumtibus fratrum Borntraeger, Königsberg, 1829, 189 pp.

[30] К. Якоби, Лекиии по динамике, ОНТИ. Гл. ред. общетехн. лит., М.-Л., 1936, 272 с.; пер. с нем.: C. G. J. Jacobi, Vorlesungen über Dynamic, Gesammelte Werke, Supplementband, G. Reimer, Berlin, 1884, 290 pp.

[31] B. Jovanović, V. Jovanović, "Geodesic and billiard flows on quadrics in pseudoEuclidean spaces: L-A pairs and Chasles theorem", Int. Math. Res. Not. IMRN (to appear).

[32] B. Khesin, S. Tabachnikov, "Pseudo-Riemannian geodesics and billiards", Adv. Math., 221:4 (2009), 1364-1396.

[33] J. L. King, "Three problems in search of a measure", Amer. Math. Monthly, 101:7 (1994), 609-628.

[34] В. В. Козлов, "Условия рациональности отношения эллиптических интегралов и большая теорема Понселе", Вестн. Моск. ун-та. Сер. 1. Матем., мех., 2003, № 4, 6-13; англ. пер.: V. V. Kozlov, "Rationality conditions for the ratio of elliptic integrals and the great Poncelet theorem", Moscow Univ. Math. Bull., 58:4 (2003), $1-7$.

[35] В. В. Козлов, “Динамические системы на торе с многозначными интегралами", Динамические системы и оптимизация, Сборник статей. K 70-летию со дня рождения академика Дмитрия Викторовича Аносова, Тр. МИАН, 256, Наука, M., 2007, 201-218; англ. пер.: V. V. Kozlov, "Dynamical systems with multivalued integrals on a torus", Proc. Steklov Inst. Math., 256:1 (2007), 188-205. 
[36] В.В. Козлов, Д.В. Трещёв, Биллиарди. Генетическое введение в динамику систем с ударами, Изд-во МГУ, М., 1991, 168 с.; англ. пер.: V. V. Kozlov, D. V. Treshchëv, Billiards. A genetic introduction to the dynamics of systems with impacts, Transl. Math. Monogr., 89, Amer. Math. Soc., Providence, RI, 1991, viii+171 pp.

[37] А. Майер, "О траекториях на ориентируемых поверхностях", Матем. сб., 12(54):1 (1943), 71-84.

[38] H. Masur, S. Tabachnikov, "Rational billiards and flat structures", Handbook of dynamical systems, v. 1A, North-Holland, Amsterdam, 2002, 1015-1089.

[39] С. П. Новиков, "Гамильтонов формализм и многозначный аналог теории Морса", УМH, 37:5(227) (1982), 3-49; англ. пер.: S. P. Novikov, "The Hamiltonian formalism and a multivalued analogue of Morse theory", Russian Math. Surveys, 37:5 (1982), $1-56$.

[40] E. Previato, "Some integrable billiards", SPT2002: Symmetry and perturbation theory (Cala Gonone), eds. S. Abenda, G. Gaeta, S. Walcher, World Sci. Publ., River Edge, NJ, 2003, 181-195.

[41] P. J. Richens, M. V. Berry, "Pseudointegrable systems in classical and quantum mechanics", Phys. D, 2:3 (1981), 495-512.

[42] P. Samuel, Projective geometry, Undergrad. Texts Math., Springer-Verlag, New York, 1988, x+156 pp.

[43] S. Tabachnikov, Geometry and billiards, Stud. Math. Libr., 30, Amer. Math. Soc., Providence, RI; Mathematics Advanced Study Semesters, University Park, PA, 2005, xii+176 pp.

[44] S. Troubetzkoy, "Typical recurrence for the Ehrenfest wind-tree model", J. Statist. Phys., 141:1 (2010), 60-67.

[45] W. A. Veech, "Strict ergodicity in zero dimensional dynamical systems and the Kronecker-Weyl theorem mod 2", Trans. Amer. Math. Soc., 140 (1969), 1-33.

[46] M. Viana, Dynamics of interval exchange transformations and Teichmüller flows, Lecture notes, 2008, 227 pp., http://w3.impa.br/ ${ }^{\sim}$ viana/out/ietf.pdf.

[47] А. Н. Земляков, А. Б. Каток, “Топологическая транзитивность биллиардов в многоугольниках", Матем. заметки, 18:2 (1975), 291-300; англ. пер.: A. N. Zemlyakov, A. B. Katok, "Topological transitivity of billiards in polygons", Math. Notes, 18:2 (1975), 760-764.

[48] A. Zorich, "Flat surfaces", Frontiers in number theory, physics and geometry, v. 1, eds. P. Cartier, B. Julia, P. Moussa, P. Vanhove, Springer, Berlin, 2006, 437-583.

\section{Владимир Ильич Драгович}

(Vladimir I. Dragović)

University of Texas at Dallas, Richardson, TX, USA;

Mathematical Institute, Serbian Academy of Sciences

and Arts, Belgrade, Serbia

E-mail: vladimir.dragovic@utdallas.edu

\section{Милена Раднович}

\section{(Milena Radnović)}

School of Mathematics and Statistics,

University of Sydney, Australia;

Mathematical Institute, Serbian Academy of Sciences

and Arts, Belgrade, Serbia

E-mail: milena.radnovic@sydney.edu.au,

milena@mi.sanu.ac.rs 